\title{
Geometry of invariant domains in complex semi-simple Lie groups
}

\author{
Christian MiebaCH
}

\begin{abstract}
We investigate the joint action of two real forms of a semi-simple complex Lie group $U^{\mathbb{C}}$ by left and right multiplication. After analyzing the orbit structure, we study the CR structure of closed orbits. The main results are an explicit formula of the Levi form of closed orbits and the determination of the Levi cone of generic orbits. Finally, we apply these results to prove $q$-completeness of certain invariant domains in $U^{\mathbb{C}}$.
\end{abstract}

Mathematics Subject Classification (2000): 22E46 (primary); 32V40 (secondary).

\section{Introduction}

Let $U^{\mathbb{C}}$ be a connected semi-simple complex Lie group with compact real form $U$ which is given by the Cartan involution $\theta$. Let us assume that there are two antiholomorphic involutive automorphisms $\sigma_{1}$ and $\sigma_{2}$ of $U^{\mathbb{C}}$ which both commute with $\theta$ and let $G_{j}=\operatorname{Fix}\left(\sigma_{j}\right), j=1,2$, be the corresponding real forms of $U^{\mathbb{C}}$. The group $G_{1} \times G_{2}$ acts on $U^{\mathbb{C}}$ by $\left(g_{1}, g_{2}\right) \cdot z:=g_{1} z g_{2}^{-1}$. In this paper we investigate complex-analytic properties of certain $\left(G_{1} \times G_{2}\right)$-invariant domains in $U^{\mathbb{C}}$ through the intrinsic Levi form of closed $\left(G_{1} \times G_{2}\right)$-orbits.

If $\sigma_{1}=\sigma_{2}=\theta$, then we discuss the $(U \times U)$-action on $U^{\mathbb{C}}$ by left and right multiplication. If $\mathfrak{t}$ is a maximal torus in $\mathfrak{u}$, then every $(U \times U)$-orbit intersects the set $\exp (i \mathfrak{t})$ in an orbit of the Weyl group $W:=\mathcal{N}_{U}(\mathfrak{t}) / \mathcal{Z}_{U}(\mathfrak{t})$. In [18] Lassalle showed that every bi-invariant domain $\Omega \subset U^{\mathbb{C}}$ is of the form $U \exp (i \omega) U$ for a $W$-invariant domain $\omega \subset \mathfrak{t}$ and that $\Omega$ is a domain of holomorphy if and only if $\omega$ is convex. In [2] Azad and Loeb proved the stronger statement that a $(U \times U)$-invariant function $\Phi$ on $\Omega$ is plurisubharmonic if and only if the $W$-invariant function

$$
\varphi: \mathfrak{t} \rightarrow \mathbb{R}, \quad \varphi(\eta):=\Phi(\exp (i \eta)),
$$

is convex.

In the case that $\sigma_{1}=\sigma_{2}$ and $G_{1}=G_{2}=: G$ is of Hermitian type, there is a distinguished $(G \times G)$-invariant in $U^{\mathbb{C}}=G^{\mathbb{C}}$, namely the open complex Ol'shanskiǔ 
semi-group. According to a result of Neeb ([23]) the open Ol'shanskiř semi-group is a domain of holomorphy.

Although the above results are statements about complex-analytic properties of domains in complex Stein manifolds, the method of their proofs is representationtheoretic. A different approach to the study of $(G \times G)$-invariant domains in $U^{\mathbb{C}}$ by analytic methods was made by Fels and Geatti in [10]. There, Fels and Geatti gave explicit formulas for the intrinsic Levi form of a closed orbit $M_{z}:=(G \times G) \cdot z$ of maximal dimension in $U^{\mathbb{C}}$ (in the following called a generic orbit) and determined the Levi cone of $M_{z}$, which enabled them to decide whether or not there may exist a bi-invariant domain of holomorphy containing $z$ in its boundary.

The main results in this paper are an explicit formula for the intrinsic Levi form of an arbitrary closed $\left(G_{1} \times G_{2}\right)$-orbit in $U^{\mathbb{C}}$ and the determination of the Levi cone of a generic orbit. We use a theorem of Matsuki [19] in order to obtain a parameterization of closed $\left(G_{1} \times G_{2}\right)$-orbits by certain Cartan algebras in the Lie algebra $\mathfrak{u}^{\mathbb{C}}=\operatorname{Lie}\left(U^{\mathbb{C}}\right)$. More precisely, there are finitely many Cartan algebras $\mathfrak{c}_{j}$ such that the closed orbits are precisely those intersecting a set of the form $C_{j}=$ $n \exp \left(i \mathfrak{c}_{j}\right)$, where the element $n$ can be chosen from a fixed torus in $U$. It turns out that the weight space decomposition of $\mathfrak{u}^{\mathbb{C}}$ with respect to $\mathfrak{c}_{j}$ is well-suited to describe the $\mathrm{CR}$ structure of closed orbits intersecting $C_{j}$. In particular, the complex tangent space of such an orbit can be identified with a direct sum of weight spaces and the intrinsic Levi form of a closed orbit is determined by the Lie bracket of certain weight vectors together with a coefficient which depends on the intersection of the orbit with $C_{j}$ (Theorem 3.15). From this fact it can be derived that the CR structures of closed orbits which belong to the same set $C_{j}$ have very similar properties.

The method used here for the derivation of explicit formulas for the Levi form is different from the one used in [10]. While Fels and Geatti found explicit local extensions of complex tangent vectors to $\mathrm{CR}$ vector fields on a generic orbit and computed their Lie brackets, the approach used here avoids these technical difficulties by pulling back the CR structure of the orbit into the Lie algebra of $G_{1} \times G_{2}$ where the Levi form can be determined via Lie-theoretic methods. In particular, we obtain a new proof for their results in the case $G_{1}=G_{2}$.

A finer analysis of the weight space decomposition of $\mathfrak{u}^{\mathbb{C}}$ with respect to $\mathfrak{c}_{j}$ reveals that it has properties very close to a root space decomposition. The most important one is the existence of $\mathfrak{s l}$ (2)-triples which enables us to determine the Levi cone of generic orbits by essentially the same method as in [10] (Theorem 3.23).

In Section 4 we give several applications of the results obtained so far. First we use the knowledge of the Levi cone in order to decide which $\left(G_{1} \times G_{2}\right)$-invariant domains containing a generic orbit in their boundary can be Stein. Secondly, we classify and study the rank one case in some detail since this case provides a class of examples where the methods and results become most transparent. This is due to the facts that complex-analytic properties of smooth domains in Stein manifolds are determined by the classical Levi form of their boundaries and that in the rank one case the boundaries of almost all invariant domains coincide with orbits of hy- 
persurface type. Finally, we define for certain $U^{\mathbb{C}}, \sigma_{1}$ and $\sigma_{2}$ a $\left(G_{1} \times G_{2}\right)$-invariant domain $\Omega \subset U^{\mathbb{C}}$ which is the right analogue of the open complex Ol'shanskir semi-group in the case $G_{1} \neq G_{2}$. We prove that the classical Levi form of a $\left(G_{1} \times G_{2}\right)$-invariant smooth function at a point $z \in \Omega$ splits into a contribution coming from the complex tangent space of $\left(G_{1} \times G_{2}\right) \cdot z$ and a contribution due to a transversal slice. Via this splitting we construct a strictly $q$-convex function on $\Omega$ which goes to infinity at $\partial \Omega$, and hence conclude that $\Omega$ is $q$-complete. However, it should be noted that the necessary conditions on $U^{\mathbb{C}}, \sigma_{1}$ and $\sigma_{2}$ are quite restrictive so that in most cases there is no non-trivial $\left(G_{1} \times G_{2}\right)$-invariant $q$-complete domain in $U^{\mathbb{C}}$.

\section{Notation}

If $\varphi$ is an automorphism of a Lie group $G$, then by abuse of notation we write $\varphi$ also for the derived automorphism of $\mathfrak{g}=\operatorname{Lie}(G)$.

ACKNOwLEDGEMENTs. This paper is a modified version of my Ph.D. thesis [21]. The support by a Promotionsstipendium of the Studienstiftung des deutschen Volkes and by SFB/TR 12 of the DFG is gratefully acknowledged. I would also like to thank the referee for his or her useful comments.

\section{The $\left(G_{1} \times G_{2}\right)$-Action on $U^{\mathbb{C}}$}

\subsection{Compatible real forms}

Let $U$ be a connected semi-simple compact Lie group. Then its universal complexification $U^{\mathbb{C}}$ is a connected semi-simple complex Lie group, and hence carries a unique structure of a linear algebraic group (compare [6]). The map $\Phi: U \times$ $i \mathfrak{u} \rightarrow U^{\mathbb{C}},(u, \xi) \mapsto u \exp (\xi)$, is a real-analytic diffeomorphism, called the Car$\tan$ decomposition of $U^{\mathbb{C}}$. Furthermore, the map $\theta: U^{\mathbb{C}} \rightarrow U^{\mathbb{C}}, \theta(u \exp (\xi)):=$ $u \exp (-\xi)$, is an anti-holomorphic involutive automorphism with $U=\operatorname{Fix}(\theta)$, called the Cartan involution of $U^{\mathbb{C}}$ corresponding to the compact real form $U$. Proofs of these facts can be found e.g. in [17].

Let $\sigma_{1}$ and $\sigma_{2}$ be two anti-holomorphic involutive automorphisms of $U^{\mathbb{C}}$, which both commute with the Cartan involution $\theta$. The fixed point set $G_{j}:=$ Fix $\left(\sigma_{j}\right)$ is a real form of $U^{\mathbb{C}}$ for $j=1,2$. The assumption that $\sigma_{j}$ commutes with $\theta$ implies that the Cartan decomposition of $U^{\mathbb{C}}$ restricts to a real-analytic diffeomorphism $K_{j} \times \mathfrak{p}_{j} \rightarrow G_{j}$, where $K_{j}:=G_{j} \cap U$ and $\mathfrak{p}_{j}:=\mathfrak{g}_{j} \cap i \mathfrak{u}$ hold. Thus the real form $G_{j}$ is a compatible subgroup of $U^{\mathbb{C}}$ in the sense of [13]. In particular, $K_{j}$ is a deformation retract of $G_{j}$.

Remark 2.1. Since $G_{j}$ is closed, the group $K_{j}$ is compact and hence a maximal compact subgroup of $G_{j}$. Thus $G_{j}$ has only finitely many connected components. 
If the group $U^{\mathbb{C}}$ is simply-connected, it follows from [26] that $G_{j}$ is connected.

The product group $G_{1} \times G_{2}$ acts on $U^{\mathbb{C}}$ by left and right multiplication, i.e. we define

$$
\left(g_{1}, g_{2}\right) \cdot z:=g_{1} z g_{2}^{-1}
$$

where $g_{j} \in G_{j}$ and $z \in U^{\mathbb{C}}$.

Definition 2.2. We say that an element $z \in U^{\mathbb{C}}$ is regular (with respect to $G_{1} \times G_{2}$ ) if the orbit $\left(G_{1} \times G_{2}\right) \cdot z$ has maximal dimension. The element $z$ is called strongly regular (with respect to $\left.G_{1} \times G_{2}\right)$, if it is regular and if $\left(G_{1} \times G_{2}\right) \cdot z$ is closed. We write $U_{r}^{\mathbb{C}}$ and $U_{s r}^{\mathbb{C}}$ for the sets of regular and strongly regular elements, respectively. Finally, we call the orbit $\left(G_{1} \times G_{2}\right) \cdot z$ generic if $z$ is strongly regular.

\section{Remark 2.3.}

(a) If we consider the action of $U^{\mathbb{C}}$ on itself given by conjugation, then Definition 2.2 yields the usual notion of (strongly) regular elements in linear algebraic groups (compare [14]).

(b) The subsets $U_{r}^{\mathbb{C}}$ and $U_{s r}^{\mathbb{C}}$ are invariant under $G_{1} \times G_{2}$. The set $U_{s r}^{\mathbb{C}}$ can be proven to be open and dense in $U^{\mathbb{C}}$ which justifies the terminology "generic orbit".

(c) In [19] an element $z \in U^{\mathbb{C}}$ is called regular semi-simple if the automorphism $\operatorname{Ad}\left(z^{-1}\right) \sigma_{1} \operatorname{Ad}(z) \sigma_{2}$ is semi-simple and if the Lie algebra $\mathfrak{g}_{2} \cap \operatorname{Ad}\left(z^{-1}\right) \mathfrak{g}_{1}$ is Abelian. It can be shown that an element is regular semi-simple in Matsuki's sense if and only if it is strongly regular.

\subsection{The isotropy representation}

The following proposition is crucial. For convenience of the reader we give a sketch of proof which makes use of the complex-analytic structure of $U^{\mathbb{C}}$.

Proposition 2.4. Let $z \in U^{\mathbb{C}}$ be a point such that the orbit $M_{z}:=\left(G_{1} \times G_{2}\right) \cdot z$ is closed. Then the isotropy group $\left(G_{1} \times G_{2}\right)_{z}$ is real-reductive and the isotropy representation of $\left(G_{1} \times G_{2}\right)_{z}$ on $T_{z} U^{\mathbb{C}}$ is completely reducible.

Proof. Since $U^{\mathbb{C}}$ is a Stein manifold, there exists a smooth strictly plurisubharmonic exhaustion function $\rho: U^{\mathbb{C}} \rightarrow \mathbb{R}$. By compactness of $U$ we can average $\rho$ using the Haar measure and hence assume that $\rho$ is $(U \times U)$-invariant. It follows that $\omega:=i \partial \bar{\partial} \rho$ is a $(U \times U)$-invariant Kähler form on $U^{\mathbb{C}}$ with respect to which $U \times U$ acts in a Hamiltonian fashion. The last statement means that there exists a $(U \times U)$-equivariant momentum map $\mu: U^{\mathbb{C}} \rightarrow \mathfrak{u}^{*} \oplus \mathfrak{u}^{*}$. Since the group $G_{1} \times G_{2}$ is compatible with the Cartan decomposition of $U^{\mathbb{C}} \times U^{\mathbb{C}}$, we can restrict $\mu$ to the subspace $\left(i \mathfrak{p}_{1}\right)^{*} \oplus\left(i \mathfrak{p}_{2}\right)^{*}$ and obtain the restricted momentum map $\mu_{i \mathfrak{p}}: U^{\mathbb{C}} \rightarrow\left(i \mathfrak{p}_{1}\right)^{*} \oplus\left(i \mathfrak{p}_{2}\right)^{*}$. According to [13] this restricted momentum map encodes a lot of information about the $\left(G_{1} \times G_{2}\right)$-action on $U^{\mathbb{C}}$ from which we need the following.

(a) $\mathrm{A}\left(G_{1} \times G_{2}\right)$-orbit is closed in $U^{\mathbb{C}}$ if and only if it intersects $\mathcal{M}_{i \mathfrak{p}}:=\mu_{i \mathfrak{p}}^{-1}(0)$ non-trivially [13, Proposition 11.2]. 
(b) If $z \in \mathcal{M}_{i \mathfrak{p}}$, then the isotropy group $\left(G_{1} \times G_{2}\right)_{z}$ is a compatible subgroup of $U^{\mathbb{C}} \times U^{\mathbb{C}}$ and hence real-reductive [13, Lemma 5.5]. Together with the previous statement this implies that isotropy groups of closed orbits are real-reductive.

(c) If $z \in \mathcal{M}_{i \mathfrak{p}}$, then the isotropy representation is completely reducible [13, Corollary 14.9].

Hence, the proposition is proven.

In the rest of this subsection we will have a closer look at the isotropy representation. Every element $\left(\xi_{1}, \xi_{2}\right) \in \mathfrak{g}_{1} \oplus \mathfrak{g}_{2}$ induces the tangent vector

$$
\begin{aligned}
\left.\frac{d}{d t}\right|_{0}\left(\exp \left(t \xi_{1}\right) z \exp \left(-t \xi_{2}\right)\right) & =\left.\frac{d}{d t}\right|_{0}\left(z \exp \left(t \operatorname{Ad}\left(z^{-1}\right) \xi_{1}\right) \exp \left(-t \xi_{2}\right)\right) \\
& =\left(\ell_{z}\right)_{*}\left(\operatorname{Ad}\left(z^{-1}\right) \xi_{1}-\xi_{2}\right) \in T_{z} U^{\mathbb{C}},
\end{aligned}
$$

where $\ell_{z}$ denotes left multiplication with $z \in U^{\mathbb{C}}$. These tangent vectors span the tangent space of the $\left(G_{1} \times G_{2}\right)$-orbit through $z$, i.e. we obtain $T_{z} M_{z}=\left(\mathfrak{g}_{1} \oplus \mathfrak{g}_{2}\right) \cdot z=$ $\left\{\left(\ell_{z}\right)_{*} \xi ; \xi \in \mathfrak{g}_{2}+\operatorname{Ad}\left(z^{-1}\right) \mathfrak{g}_{1}\right\}$.

Let $\rho$ denote the isotropy representation of $\left(G_{1} \times G_{2}\right)_{z}$ on $T_{z} U^{\mathbb{C}}$. One checks directly that the isotropy group at $z \in U^{\mathbb{C}}$ is given by

$$
\left(G_{1} \times G_{2}\right)_{z}=\left\{\left(z g_{2} z^{-1}, g_{2}\right) ; g_{2} \in G_{2} \cap z^{-1} G_{1} z\right\} .
$$

Consequently, we may identify $\left(G_{1} \times G_{2}\right)_{z}$ with $G_{2} \cap z^{-1} G_{1} z$ via the isomorphism $\Phi: G_{2} \cap z^{-1} G_{1} z \rightarrow\left(G_{1} \times G_{2}\right)_{z}, g \mapsto\left(z g z^{-1}, g\right)$. Similarly, we will identify the tangent space $T_{z} M_{z}$ with $\mathfrak{g}_{2}+\operatorname{Ad}\left(z^{-1}\right) \mathfrak{g}_{1}$ via $\left(\ell_{z}\right)_{*}$. We conclude from

$$
\begin{aligned}
\rho(\Phi(g))\left(\ell_{z}\right)_{*} \xi & =\left.\frac{d}{d t}\right|_{0}\left(z g z^{-1}, g\right) \cdot(z \exp (t \xi)) \\
& =\left.\frac{d}{d t}\right|_{0}\left(z g \exp (t \xi) g^{-1}\right) \\
& =\left.\frac{d}{d t}\right|_{0} z \exp (t \operatorname{Ad}(g) \xi)=\left(\ell_{z}\right)_{*} \operatorname{Ad}(g) \xi
\end{aligned}
$$

that the map $\left(\ell_{z}\right)_{*}$ intertwines the adjoint representation of $G_{2} \cap z^{-1} G_{1} z$ on $\mathfrak{u}^{\mathbb{C}}$ with the isotropy representation of $\left(G_{1} \times G_{2}\right)_{z}$ on $T_{z} U^{\mathbb{C}}$ modulo $\Phi$. We summarize our considerations in the following:

Proposition 2.5. Modulo the isomorphism $\Phi$ the isotropy representation of $\left(G_{1} \times\right.$ $\left.G_{2}\right)_{z}$ on $T_{z} U^{\mathbb{C}}$ is equivalent to the adjoint representation of $G_{2} \cap z^{-1} G_{1} z$ on $\mathfrak{u}^{\mathbb{C}}$. 


\subsection{The orbit structure theorem}

We review the main results of [19] in order to describe the orbit structure of the $\left(G_{1} \times G_{2}\right)$-action on $U^{\mathbb{C}}$. A proof of Matsuki's theorem which relies on the momentum map techniques developed in [13] can be found in [21].

Let $\mathfrak{a}_{0}$ be a maximal Abelian subspace of $\mathfrak{p}_{1} \cap \mathfrak{p}_{2}$ and let $\mathfrak{t}_{0}$ be a maximal torus in the centralizer of $\mathfrak{a}_{0}$ in $\mathfrak{k}_{1} \cap \mathfrak{k}_{2}$. It follows that $\mathfrak{c}_{0}:=\mathfrak{t}_{0} \oplus \mathfrak{a}_{0}$ is a maximally non-compact $\theta$-invariant Cartan subalgebra of $\mathfrak{g}_{1} \cap \mathfrak{g}_{2}$.

Remark 2.6. By maximality of $\mathfrak{a}_{0}$ the group $A_{0}^{c}:=\exp \left(i \mathfrak{a}_{0}\right)$ is a compact torus in $U$.

Definition 2.7. A subset of the form $C=n \exp (i \mathfrak{c}) \subset U^{\mathbb{C}}$ is called a standard Cartan subset, if $n \in A_{0}^{c}$ and $\mathfrak{c}=\mathfrak{t} \oplus \mathfrak{a}$ is a $\theta$-stable Cartan subalgebra of $\mathfrak{g}_{2} \cap$ $\operatorname{Ad}\left(n^{-1}\right) \mathfrak{g}_{1}$ such that $\mathfrak{t} \supset \mathfrak{t}_{0}, \mathfrak{a} \subset \mathfrak{a}_{0}$ and $\operatorname{dim} \mathfrak{c}=\operatorname{dim} \mathfrak{c}_{0}$ hold. The standard Cartan subset $C_{0}:=\exp \left(i \mathfrak{c}_{0}\right)$ is called the fundamental Cartan subset.

We call two standard Cartan subsets equivalent if there is a generic $\left(G_{1} \times\right.$ $G_{2}$ )-orbit which intersects both non-trivially. Let $\left\{C_{j}\right\}_{j \in J}$ be a complete set of representatives for the equivalence classes. For each $j \in J$ we define the groups

$$
\begin{aligned}
\mathcal{N}_{K_{1} \times K_{2}}\left(C_{j}\right) & :=\left\{\left(k_{1}, k_{2}\right) \in K_{1} \times K_{2} ; k_{1} C_{j} k_{2}^{-1}=C_{j}\right\}, \\
\mathcal{Z}_{K_{1} \times K_{2}}\left(C_{j}\right) & :=\left\{\left(k_{1}, k_{2}\right) \in K_{1} \times K_{2} ; k_{1} z k_{2}^{-1}=z \text { for all } z \in C_{j}\right\},
\end{aligned}
$$

and $W_{K_{1} \times K_{2}}\left(C_{j}\right):=\mathcal{N}_{K_{1} \times K_{2}}\left(C_{j}\right) / \mathcal{Z}_{K_{1} \times K_{2}}\left(C_{j}\right)$.

Remark 2.8. The group $W_{K_{1} \times K_{2}}\left(C_{j}\right)$ is finite for each $j \in J$.

Theorem 2.9 (Matsuki). The set $J$ is finite and we have

$$
U_{c l}^{\mathbb{C}}=\bigcup_{j \in J} G_{1} C_{j} G_{2} \quad \text { and } \quad U_{s r}^{\mathbb{C}}=\bigcup_{j \in J} G_{1}\left(C_{j} \cap U_{s r}^{\mathbb{C}}\right) G_{2},
$$

where $U_{c l}^{\mathbb{C}}:=\left\{z \in U^{\mathbb{C}} ;\left(G_{1} \times G_{2}\right) \cdot z\right.$ is closed $\}$. Moreover, each generic $\left(G_{1} \times G_{2}\right)$ orbit intersects $C_{j}$ in a $W_{K_{1} \times K_{2}}\left(C_{j}\right)$-orbit.

Remark 2.10. If $G_{1}=G_{2}$, then let $\mathfrak{c}_{0}, \ldots, \mathfrak{c}_{k}$ be a complete set of representatives for the equivalence classes of Cartan subalgebras of $\mathfrak{g}_{1}$. We can assume without loss of generality that each $\mathfrak{c}_{j}$ is $\theta$-stable. Let $\left\{n_{j, l}\right\} \subset \mathcal{N}_{U^{\mathbb{C}}}\left(\mathfrak{c}_{l}\right)$ be a complete set of representatives for the double cosets $W_{G_{1}}\left(\mathfrak{c}_{l}\right) \backslash W_{U^{\mathbb{C}}}\left(\mathfrak{c}_{l}\right) / W_{G_{1}}\left(\mathfrak{c}_{l}\right)$. It can be shown that the sets $n_{j, l} \exp \left(i \mathfrak{c}_{j}\right)$ exhaust the equivalence classes of standard Cartan subsets for the $\left(G_{1} \times G_{1}\right)$-action on $U^{\mathbb{C}}$. This is the content of a theorem of Bremigan ([5], see [10] for the formulation given here).

\subsection{The weight space decomposition}

Let $C=n \exp (i \mathfrak{c})$ be a standard Cartan subset. In this subsection we discuss the weight space decomposition

$$
\mathfrak{u}^{\mathbb{C}}=\bigoplus_{\lambda \in \Lambda} \mathfrak{u}_{\lambda}^{\mathbb{C}}
$$


of $\mathfrak{u}^{\mathbb{C}}$ with respect to the Cartan subalgebra $\mathfrak{c} \subset \mathfrak{g}_{2} \cap \operatorname{Ad}\left(n^{-1}\right) \mathfrak{g}_{1}$. Here, we have written $\Lambda=\Lambda\left(\mathfrak{u}^{\mathbb{C}}, \mathfrak{c}\right)$ for the set of weights and $\mathfrak{u}_{\lambda}^{\mathbb{C}}$ for the weight space corresponding to the weight $\lambda$. We say that the weight $\lambda$ is real (respectively imaginary) if $\lambda \neq 0$ and $\lambda(\mathfrak{c}) \subset \mathbb{R}$ (respectively $\lambda(\mathfrak{c}) \subset i \mathbb{R}$ ) holds. A non-zero weight which is neither real nor imaginary is called complex. We write $\Lambda_{r}, \Lambda_{i}$ and $\Lambda_{c}$ for the sets of real, imaginary and complex weights, and obtain

$$
\Lambda \backslash\{0\}=\Lambda_{r} \dot{\cup} \Lambda_{i} \dot{\cup} \Lambda_{c} .
$$

Remark 2.11. We extend the weight $\lambda$ by $\mathbb{C}$-linearity to the complexified Cartan algebra $\mathfrak{c}^{\mathbb{C}}$. Since $\lambda(\mathfrak{t}) \subset i \mathbb{R}$ and $\lambda(\mathfrak{a}) \subset \mathbb{R}$ hold for all $\lambda \in \Lambda$, we conclude that the weights are real-valued on $i \mathfrak{t} \oplus \mathfrak{a}$.

Since $n \in A_{0}^{c}$, the automorphism $\tau_{n}:=\operatorname{Ad}\left(n^{-1}\right) \sigma_{1} \operatorname{Ad}(n) \sigma_{2} \in \operatorname{Aut}\left(\mathfrak{u}^{\mathbb{C}}\right)$ is unitary with respect to the Hermitian inner product $\left\langle\xi_{1}, \xi_{2}\right\rangle:=-B_{\mathfrak{u}^{\mathbb{C}}}\left(\xi_{1}, \theta\left(\xi_{2}\right)\right)$, where $B_{\mathfrak{u}^{\mathbb{C}}}$ is the Killing form of $\mathfrak{u}^{\mathbb{C}}$. Consequently, $\tau_{n}$ is semi-simple with eigenvalues in the unit circle $S^{1}$. Since $\tau_{n}$ leaves $\mathfrak{c}$ pointwise fixed, each weight space $\mathfrak{u}_{\lambda}^{\mathbb{C}}$ is invariant under $\tau_{n}$. Hence, following [19] we obtain the finer decomposition

$$
\mathfrak{u}^{\mathbb{C}}=\bigoplus_{(\lambda, a) \in \tilde{\Lambda}} \mathfrak{u}_{\lambda, a}^{\mathbb{C}},
$$

where $\mathfrak{u}_{\lambda, a}^{\mathbb{C}}:=\left\{\xi \in \mathfrak{u}_{\lambda}^{\mathbb{C}} ; \tau_{n}(\xi)=a \xi\right\}$ and $\tilde{\Lambda}:=\left\{(\lambda, a) \in \Lambda \times S^{1} ; \mathfrak{u}_{\lambda, a}^{\mathbb{C}} \neq\{0\}\right\}$. The elements of $\widetilde{\Lambda}$ are called the extended weights, and (2.2) is called the extended weight space decomposition.

Remark 2.12. Since $\mathfrak{c}$ is a Cartan subalgebra of $\mathfrak{g}_{2} \cap \operatorname{Ad}\left(n^{-1}\right) \mathfrak{g}_{1}$, we conclude $\mathfrak{u}_{0,1}^{\mathbb{C}}=\mathfrak{c}^{\mathbb{C}}$. Moreover, for the fundamental Cartan subspace $\mathfrak{c}_{0} \subset \mathfrak{g}_{1} \cap \mathfrak{g}_{2}$ we have $\left(\mathfrak{g}_{1} \cap \mathfrak{g}_{2}\right)^{\mathbb{C}}=\mathfrak{c}^{\mathbb{C}} \oplus \bigoplus_{(\lambda, 1) \in \widetilde{\Lambda}} \mathfrak{u}_{\lambda, 1}^{\mathbb{C}}$.

We collect some properties of the extended weight space decomposition in the following

\section{Lemma 2.13.}

(1) The Cartan involution $\theta$ maps $\mathfrak{u}_{\lambda, a}^{\mathbb{C}}$ onto $\mathfrak{u}_{-\lambda, a^{-1}}^{\mathbb{C}}$. In particular, if $(\lambda, a)$ is an extended weight, then $\left(-\lambda, a^{-1}\right)$ is an extended weight, too.

(2) We have $B_{\mathfrak{u}^{\mathbb{C}}}\left(\mathfrak{u}_{\lambda, a}^{\mathbb{C}}, \mathfrak{u}_{\mu, b}^{\mathbb{C}}\right)=0$ unless $(\lambda, a)=\left(-\mu, b^{-1}\right) \in \widetilde{\Lambda}$.

(3) Let $\xi_{\lambda, a} \in \mathfrak{u}_{\lambda, a}^{\mathbb{C}}$ with $\left\|\xi_{\lambda, a}\right\|=1$ be given and let $\eta_{\lambda, a}:=-\left[\xi_{\lambda, a}, \theta\left(\xi_{\lambda, a}\right)\right]$. Then we have $B_{\mathfrak{u}^{\mathbb{C}}}\left(\eta_{\lambda, a}, \eta\right)=\lambda(\eta)$ for all $\eta \in \mathfrak{c}$. In particular, $\eta_{\lambda, a}$ does not depend on the element $a \in S^{1}$, i.e. $\eta_{\lambda, a}=\eta_{\lambda, a^{\prime}}=: \eta_{\lambda}$ for all $(\lambda, a),\left(\lambda, a^{\prime}\right) \in \widetilde{\Lambda}$.

(4) We have $\left[\xi_{\lambda, a}, \xi\right]=B_{\mathfrak{u}^{\mathbb{C}}}\left(\xi_{\lambda, a}, \xi\right) \eta_{\lambda}$ for all $\xi \in \mathfrak{u}_{-\lambda, a^{-1}}^{\mathbb{C}}$.

Proof. In order to prove the first claim let $\eta=\eta_{\mathfrak{t}}+\eta_{\mathfrak{a}} \in \mathfrak{t} \oplus \mathfrak{a}=\mathfrak{c}$ and $\xi \in \mathfrak{u}_{\alpha, \lambda}^{\mathbb{C}}$ be given and consider

$$
[\eta, \theta(\xi)]=\theta[\theta(\eta), \xi]=\theta\left[\eta_{\mathfrak{t}}-\eta_{\mathfrak{a}}, \xi\right]=\theta\left(\lambda\left(\eta_{\mathfrak{t}}\right) \xi\right)-\theta\left(\lambda\left(\eta_{\mathfrak{a}}\right) \xi\right)=-\lambda(\eta) \theta(\xi) .
$$


Here we used the facts that $\lambda(\mathfrak{t}) \subset i \mathbb{R}$ while $\lambda(\mathfrak{a}) \subset \mathbb{R}$ and that $\theta$ is $\mathbb{C}$-anti-linear. Since $\theta$ commutes with $\tau_{n}$, we conclude

$$
\tau_{n} \theta(\xi)=\theta \tau_{n}(\xi)=\theta(a \xi)=\bar{a} \theta(\xi)=a^{-1} \theta(\xi),
$$

which proves the first claim.

The second claim follows from the fact that the Killing form $B_{\mathfrak{u}^{\mathbb{C}}}$ is invariant under $\operatorname{Aut}\left(\mathfrak{u}^{\mathbb{C}}\right)$.

In order to prove the third one we compute

$$
\begin{aligned}
B_{\mathfrak{u}^{\mathbb{C}}}\left(\eta_{\lambda, a}, \eta\right)=-B_{\mathfrak{u}^{\mathbb{C}}}\left(\left[\xi_{\lambda, a}, \theta\left(\xi_{\lambda, a}\right)\right], \eta\right) & =B_{\mathfrak{u}^{\mathbb{C}}}\left(\theta\left(\xi_{\lambda, a}\right),\left[\xi_{\lambda, a}, \eta\right]\right) \\
& =-\lambda(\eta) B_{\mathfrak{u}^{\mathbb{C}}}\left(\xi_{\lambda, a}, \theta\left(\xi_{\lambda, a}\right)\right) \\
& =\lambda(\eta)\left\|\xi_{\lambda, a}\right\|^{2}=\lambda(\eta) .
\end{aligned}
$$

The last claim is proven in the same way as in [17, Lemma 2.18(a)].

Standard arguments from Lie theory (see for example [17, Chapter II.4]) lead to the following result.

\section{Proposition 2.14.}

(1) Let $\lambda \neq 0$. After a suitable normalization the elements $\eta_{\lambda}, \xi_{\lambda, a}$ and $\theta\left(\xi_{\lambda, a}\right)$ form an $\mathfrak{s l}(2)$-triple.

(2) If $\lambda \neq 0$, then we have $\operatorname{dim}_{\mathbb{C}} \mathfrak{u}_{\lambda, a}^{\mathbb{C}}=1$ and $\operatorname{dim}_{\mathbb{C}} \mathfrak{u}_{m \lambda, a^{m}}^{\mathbb{C}}=0$ for all $m \geq 2$.

(3) The set $\Lambda \backslash\{0\}$ of non-zero weights fulfills the axioms of an abstract root system in $(i \mathfrak{t} \oplus \mathfrak{a})^{*}$.

(4) Let $\lambda, \mu \in \Lambda \backslash\{0\}$ such that $\lambda+\mu \in \Lambda \backslash\{0\}$ holds. Then we have $\left[\mathfrak{u}_{\lambda}^{\mathbb{C}}, \mathfrak{u}_{\mu}^{\mathbb{C}}\right]=$ $\mathfrak{u}_{\lambda+\mu}^{\mathbb{C}}$

\section{CR Geometry of Closed Orbits}

\subsection{Preliminaries from CR geometry}

In this subsection we will review the basic definitions and facts from the theory of CR submanifolds as far as they are needed later on. For more details and complete proofs we refer the reader to the textbooks [3] and [4].

Let $Z$ be a complex manifold with complex structure $J$. A real submanifold $M$ of $Z$ is called a Cauchy-Riemann or CR submanifold if the dimension of the complex tangent space $H_{p} M:=T_{p} M \cap J_{p} T_{p} M$ does not depend on the point $p \in$ $M$. In this case, the set $H M:=\bigcup_{p \in M} H_{p} M$ is a smooth subbundle of the tangent bundle $T M$ invariant under the complex structure $J$, called the complex tangent bundle of $M$. A CR submanifold $M \subset Z$ is called generic if $T_{p} M+J_{p} T_{p} M=T_{p} Z$ holds for all $p \in M$. For example, every smooth real hypersurface in $Z$ is a generic CR submanifold of $Z$. 
Remark 3.1. Since the group $G_{1} \times G_{2}$ acts by holomorphic transformations on $U^{\mathbb{C}}$, each closed $\left(G_{1} \times G_{2}\right)$-orbit is a $\mathrm{CR}$ submanifold of $U^{\mathbb{C}}$. Since the $\left(G_{1} \times G_{2}\right)$-action extends to a transitive $\left(U^{\mathbb{C}} \times U^{\mathbb{C}}\right)$-action on $U^{\mathbb{C}}$, each closed orbit is moreover generic as a $\mathrm{CR}$ submanifold.

A smooth section in $H M$ is called a CR vector field on $M$. A smooth map $f$ from $M$ into a CR submanifold $M^{\prime} \subset\left(Z^{\prime}, J^{\prime}\right)$ is called a CR map if $f_{*}$ maps $H M$ into $H M^{\prime}$ and if $f_{*} J=J^{\prime} f_{*}$ holds. A CR function on $M$ is a CR map $M \rightarrow \mathbb{C}$, where $\mathbb{C}$ is equipped with its usual structure as complex manifold.

For each CR submanifold $M \subset Z$ one can define the intrinsic Levi form, which generalizes the classical Levi form of a smooth hypersurface.

Definition 3.2. The Levi form of $M$ at the point $p$ is the map $\mathcal{L}_{p}: H_{p} M \times H_{p} M \rightarrow$ $T_{p}^{\mathbb{C}} M / H_{p}^{\mathbb{C}} M$ defined by

$$
\mathcal{L}_{p}(v, w):=\left(\frac{i}{2}[V, W]_{p}-\frac{1}{2}[V, J W]_{p}\right) \bmod H_{p}^{\mathbb{C}} M,
$$

where $V$ and $W$ are CR vector fields on $M$ with $V_{p}=v$ and $W_{p}=w$.

Remark 3.3. One can show that the intrinsic Levi form is well-defined, i.e. that it does not depend on the choice of CR extensions of $v, w \in H_{p} M$ (compare [4]).

The Levi cone $\mathcal{C}_{p}$ of $M$ at $p$ is by definition the closed convex cone generated by the vectors $\mathcal{L}_{p}(v, v)$ where $v$ runs through $H_{p} M$. Because of $\mathcal{L}_{p}(v, w)=$ $\overline{\mathcal{L}_{p}(w, v)}$ the Levi cone is contained in $T_{p} M / H_{p} M$. The Levi cone generalizes the signature of the classical Levi form of a hypersurface. Its significance stems from the fact that it governs the local extension of CR functions on $M$ to holomorphic functions on $Z$.

Theorem 3.4 (Boggess, Polking). Let $M$ be a generic CR submanifold of a complex manifold $Z$ and let us assume that the Levi cone at some point $p \in M$ satisfies $\mathcal{C}_{p}(M)=T_{p} M / H_{p} M$. Then, for each neighborhood $\omega$ of $p$ in $M$ there exists a neighborhood $\Omega$ of p in $Z$ satisfying $\Omega \cap M \subset \omega$ which has the property that every CR function on $\Omega \cap M$ extends to a unique holomorphic function on $\Omega$.

A proof of this theorem can be found in [4].

\subsection{The complex tangent space of a closed orbit}

Let $z \in U^{\mathbb{C}}$ be given such that the orbit $M_{z}=\left(G_{1} \times G_{2}\right) \cdot z$ is closed in $U^{\mathbb{C}}$. By Matsuki's theorem we can assume that there is a standard Cartan subset $C=$ $n \exp (i \mathfrak{c})$ which contains $z=n \exp (i \eta)$. We define

$$
\widetilde{\Lambda}(z):=\left\{(\lambda, a) \in \tilde{\Lambda} ; a e^{-2 i \lambda(\eta)}=1\right\}
$$

and set $\tau_{z}:=\operatorname{Ad}\left(z^{-1}\right) \sigma_{1} \operatorname{Ad}(z) \sigma_{2} \in \operatorname{Aut}\left(\mathfrak{u}^{\mathbb{C}}\right)$. 
Lemma 3.5. The automorphism $\tau_{z}$ is semi-simple and we have

$$
\operatorname{Fix}\left(\tau_{z}\right)=\left(\mathfrak{g}_{2} \cap \operatorname{Ad}\left(z^{-1}\right) \mathfrak{g}_{1}\right)^{\mathbb{C}}=\bigoplus_{(\lambda, a) \in \widetilde{\Lambda}(z)} \mathfrak{u}_{\lambda, a}^{\mathbb{C}}
$$

Proof. The first equality can be deduced from [19, page 57]. In order to prove the second one let $\xi=\sum_{(\lambda, a)} \xi_{\lambda, a}$ be an arbitrary element of $\mathfrak{u}^{\mathbb{C}}$. Then we have

$$
\begin{aligned}
\tau_{z}(\xi)=\operatorname{Ad}\left(z^{-1}\right) \sigma_{1} \operatorname{Ad}(z) \sigma_{2}(\xi) & =\operatorname{Ad}(\exp (-i \eta)) \tau_{n} \operatorname{Ad}(\exp (-i \eta)) \xi \\
& =\operatorname{Ad}(\exp (-i \eta)) \tau_{n}\left(\sum_{(\lambda, a)} e^{-i \lambda(\eta)} \xi_{\lambda, a}\right) \\
& =\operatorname{Ad}(\exp (-i \eta)) \sum_{(\lambda, a)} a e^{-i \lambda(\eta)} \xi_{\lambda, a} \\
& =\sum_{(\lambda, a)} a e^{-2 i \lambda(\eta)} \xi_{\lambda, a} .
\end{aligned}
$$

This proves that $\tau_{z}$ is semi-simple. Moreover, $\tau_{z}(\xi)=\xi$ holds if and only if $\xi_{\lambda, a}=0$ for all $(\lambda, a) \notin \widetilde{\Lambda}(z)$.

Since $\mathfrak{g}_{2} \cap \operatorname{Ad}\left(z^{-1}\right) \mathfrak{g}_{1}$ is isomorphic to the Lie algebra of $\left(G_{1} \times G_{2}\right)_{z}$, we obtain the following characterization of strongly regular elements in terms of the extended weights as a corollary.

Theorem 3.6. We have $\operatorname{codim}_{\mathbb{R}}\left(G_{1} \underset{\sim}{\times} G_{2}\right) \cdot z=\operatorname{dim}_{\mathbb{R}} \mathfrak{c}+(\# \tilde{\Lambda}(z)-1)$. The element $z$ is strongly regular if and only if $\widetilde{\Lambda}(z)=\{(0,1)\}$ holds. This implies that the codimension of a generic orbit coincides with the rank of the real-reductive Lie algebra $\mathfrak{g}_{1} \cap \mathfrak{g}_{2}$.

Finally we describe the tangent space $T_{z} M_{z}$ in terms of the extended weight space decomposition.

Theorem 3.7. Under the map $\left(\ell_{z}\right)_{*}$ the tangent space $T_{z} M_{z}$ is isomorphic to

$$
\mathfrak{g}_{2}+\operatorname{Ad}\left(z^{-1}\right) \mathfrak{g}_{1}=\left(\mathfrak{g}_{2} \cap \operatorname{Ad}\left(z^{-1}\right) \mathfrak{g}_{1}\right) \oplus \bigoplus_{(\lambda, a) \notin \widetilde{\Lambda}(z)} \mathfrak{u}_{\lambda, a}^{\mathbb{C}}
$$

In particular, the complex tangent space of $\left(G_{1} \times G_{2}\right) \cdot z$ is isomorphic to $\bigoplus_{(\lambda, a) \notin \widetilde{\Lambda}(z)} \mathfrak{u}_{\lambda, a}^{\mathbb{C}}$.

Remark 3.8. From now on we will identify the quotient $T_{z}^{\mathbb{C}} M / H_{z}^{\mathbb{C}} M$ with $R_{z}^{\mathbb{C}} M:=$ $\left(\ell_{z}\right)_{*}\left(\mathfrak{g}_{2} \cap \operatorname{Ad}\left(z^{-1}\right) \mathfrak{g}_{1}\right)^{\mathbb{C}}$. It follows that these spaces are isomorphic as $\left(G_{1} \times G_{2}\right)_{z^{-}}$ modules. 
Proof of Theorem 3.7. Since $\tau_{z}$ is semi-simple, we conclude from [19, Lemma 1(i)] that

$$
\mathfrak{u}^{\mathbb{C}}=i\left(\mathfrak{g}_{2} \cap \operatorname{Ad}\left(z^{-1}\right) \mathfrak{g}_{1}\right) \oplus\left(\mathfrak{g}_{2}+\operatorname{Ad}\left(z^{-1}\right) \mathfrak{g}_{1}\right)
$$

holds. Moreover, one checks directly that this decomposition is orthogonal with respect to the real part of the Killing form $B_{\mathfrak{u}^{\mathbb{C}}}$. Similarly, we have the decomposition

$$
\mathfrak{u}^{\mathbb{C}}=\operatorname{Fix}\left(\tau_{z}\right) \oplus \operatorname{Fix}\left(\tau_{z}\right)^{\perp}, \quad \operatorname{Fix}\left(\tau_{z}\right)=\left(\mathfrak{g}_{2} \cap \operatorname{Ad}\left(z^{-1}\right) \mathfrak{g}_{1}\right)^{\mathbb{C}}
$$

where the orthogonal complement $\operatorname{Fix}\left(\tau_{z}\right)^{\perp}$ with respect to $B_{\mathfrak{u}^{\mathbb{C}}}$ is the sum of the $\tau_{z}$-eigenspaces corresponding to eigenvalues $\neq 1$. These observations imply

$$
\mathfrak{g}_{2}+\operatorname{Ad}\left(z^{-1}\right) \mathfrak{g}_{1}=\left(\mathfrak{g}_{2} \cap \operatorname{Ad}\left(z^{-1}\right) \mathfrak{g}_{1}\right) \oplus \operatorname{Fix}\left(\tau_{z}\right)^{\perp} .
$$

Since the same argument as the one in the proof of Lemma 3.5 implies the equality

$$
\operatorname{Fix}\left(\tau_{z}\right)^{\perp}=\bigoplus_{(\lambda, a) \notin \widetilde{\Lambda}(z)} \mathfrak{u}_{\lambda, a}^{\mathbb{C}},
$$

the theorem is proven.

\subsection{Pulling back the Levi form into the Lie algebra}

As abbreviation we put $G:=G_{1} \times G_{2}$ in this subsection. Consequently, we have $\mathfrak{g}:=\operatorname{Lie}(G)=\mathfrak{g}_{1} \oplus \mathfrak{g}_{2}$.

As we have remarked above, every closed $G$-orbit $M_{z}=G \cdot z$ is a generic CR submanifold of $U^{\mathbb{C}}$. Let $\pi_{z}: \mathfrak{g} \rightarrow \mathfrak{g} \cdot z=T_{z} M_{z}$ be the differential of the orbit map. By Equation (2.1) the map $\pi_{z}$ is given by

$$
\pi_{z}\left(\xi_{1}, \xi_{2}\right)=\left(\ell_{z}\right)_{*}\left(\operatorname{Ad}\left(z^{-1}\right) \xi_{1}-\xi_{2}\right)
$$

In this subsection we will pull back the CR structure of $M_{z}$ into the Lie algebra $\mathfrak{g}$ and compute the Levi form of $M_{z}$ via this pull back. The following proposition is essential.

Proposition 3.9. We have the $G_{z}$-invariant decomposition $\mathfrak{g}=\mathfrak{g}_{z} \oplus \mathfrak{q}_{z}$, and $\mathfrak{q}_{z}$ and $T_{z} M_{z}$ are isomorphic as $G_{z}$-spaces where the isomorphism is given by $\tilde{\pi}_{z}:=\left.\pi_{z}\right|_{\mathfrak{q}_{z}}$. Since the complex tangent space $H_{z} M_{z}$ is invariant under $G_{z}$, we obtain the $G_{z^{-}}$ invariant decomposition $\mathfrak{q}_{z}=R\left(\mathfrak{q}_{z}\right) \oplus H\left(\mathfrak{q}_{z}\right)$ where $H\left(\mathfrak{q}_{z}\right):=\tilde{\pi}_{z}^{-1}\left(H_{z} M_{z}\right)$ and $R\left(\mathfrak{q}_{z}\right):=\tilde{\pi}_{z}^{-1}\left(R_{z} M_{z}\right)$.

Proof. We only have to show that the adjoint representation of $G_{z}$ on $\mathfrak{g}$ is completely reducible. This follows from Proposition 2.4 since $G_{z}$ is conjugate to a compatible subgroup of $U^{\mathbb{C}} \times U^{\mathbb{C}}$ if the orbit $G \cdot z=M_{z}$ is closed. 
Proposition 3.10. The Levi form $\mathcal{L}_{z}: H_{z} M_{z} \times H_{z} M_{z} \rightarrow R_{z}^{\mathbb{C}} M_{z}$ is given by $\mathcal{L}_{z}(v, w)=\pi_{z}\left(\frac{i}{2}\left[\tilde{\pi}_{z}^{-1}(v), \tilde{\pi}_{z}^{-1}(w)\right]-\frac{1}{2}\left[\tilde{\pi}_{z}^{-1}(v), \tilde{\pi}_{z}^{-1}(i w)\right]\right) \bmod H_{z}^{\mathbb{C}} M_{z}$.

Proof. Let $v, w \in H_{z} M_{z}$ be given and let $V, W$ be CR vector fields on $M_{z}$ with $V_{z}=v$ and $W_{z}=w$. Since the orbit map $G \rightarrow M_{z}$ is a $G_{z}$-principal bundle, there exist projectable vector fields $\widetilde{V}$ and $\widetilde{W}$ on $G$ with $\widetilde{V}_{e}=\widetilde{\pi}_{z}^{-1}(v)$ and $\widetilde{W}_{e}=\widetilde{\pi}_{z}^{-1}(w)$ such that $\pi_{z} \widetilde{V}=V$ and $\pi_{z} \widetilde{W}=W$ hold. For a proof of this fact and more details about projectable vector fields we refer the reader to [16]. Although it is in general not possible to choose the vector fields $\widetilde{V}$ and $\widetilde{W}$ to be left-invariant, the same argument which proves well-definedness of the intrinsic Levi form applies to show that

$$
\left(\frac{i}{2}[\widetilde{V}, \widetilde{W}]_{e}-\frac{1}{2}[\widetilde{V}, \widetilde{J W}]_{e}\right) \bmod H^{\mathbb{C}}\left(\mathfrak{q}_{z}\right)
$$

does only depend on the values $\widetilde{V}_{e}$ and $\widetilde{W}_{e}$ (compare the proof of Lemma 1 in Chapter 10.1 of [4]). Therefore we conclude

$$
\begin{aligned}
\left(\frac{i}{2}[\tilde{V}, \tilde{W}]_{e}-\frac{1}{2}[\tilde{V}, \widetilde{J W}]_{e}\right) \bmod H^{\mathbb{C}}\left(\mathfrak{q}_{z}\right) & =\left(\frac{i}{2}\left[\tilde{\pi}_{z}^{-1}(v), \tilde{\pi}_{z}^{-1}(w)\right]\right. \\
& \left.-\frac{1}{2}\left[\tilde{\pi}_{z}^{-1}(v), \tilde{\pi}_{z}^{-1}(i w)\right]\right) \bmod H^{\mathbb{C}}\left(\mathfrak{q}_{z}\right),
\end{aligned}
$$

and obtain

$$
\begin{aligned}
\mathcal{L}_{z}(v, w) & =\left(\frac{i}{2}[V, W]_{z}-\frac{1}{2}[V, J W]_{z}\right) \bmod H_{z}^{\mathbb{C}} M_{z} \\
& =\left(\frac{i}{2}\left[\pi_{z} \widetilde{V}, \pi_{z} \widetilde{W}\right]_{z}-\frac{1}{2}\left[\pi_{z} \widetilde{V}, \pi_{z} \widetilde{J W}\right]_{z}\right) \bmod H_{z}^{\mathbb{C}} M_{z} \\
& =\left(\frac{i}{2} \pi_{z}[\tilde{V}, \widetilde{W}]_{e}-\frac{1}{2} \pi_{z}[\tilde{V}, \widetilde{J W}]_{e}\right) \bmod H_{z}^{\mathbb{C}} M_{z} \\
& =\pi_{z}\left(\frac{i}{2}[\widetilde{V}, \widetilde{W}]_{e}-\frac{1}{2}[\tilde{V}, \widetilde{J W}]_{e} \bmod H^{\mathbb{C}}\left(\mathfrak{q}_{z}\right)\right) \\
& =\pi_{z}\left(\frac{i}{2}\left[\tilde{\pi}_{z}^{-1}(v), \tilde{\pi}_{z}^{-1}(w)\right]-\frac{1}{2}\left[\tilde{\pi}_{z}^{-1}(v), \tilde{\pi}_{z}^{-1}(i w)\right] \bmod H^{\mathbb{C}}\left(\mathfrak{q}_{z}\right)\right) \\
& =\pi_{z}\left(\frac{i}{2}\left[\tilde{\pi}_{z}^{-1}(v), \tilde{\pi}_{z}^{-1}(w)\right]-\frac{1}{2}\left[\tilde{\pi}_{z}^{-1}(v), \tilde{\pi}_{z}^{-1}(i w)\right]\right) \bmod H_{z}^{\mathbb{C}} M_{z} .
\end{aligned}
$$

This finishes the proof.

In the next subsection we will use the weight space decomposition in order to determine the map $\tilde{\pi}_{z}^{-1}$ explicitly. 


\subsection{The Levi form of a closed orbit}

This rather technical subsection contains the computations which are necessary to achieve the final formula of the Levi form.

Lemma 3.11. We have

$$
\mathfrak{g}_{z}=\operatorname{ker}\left(\pi_{z}\right)=\left\{(\operatorname{Ad}(z) \xi, \xi) ; \xi \in \mathfrak{g}_{2} \cap \operatorname{Ad}\left(z^{-1}\right) \mathfrak{g}_{1}\right\} .
$$

Proof. One checks directly that $\left\{(\operatorname{Ad}(z) \xi, \xi) ; \xi \in \mathfrak{g}_{2} \cap \operatorname{Ad}\left(z^{-1}\right) \mathfrak{g}_{1}\right\} \subset \operatorname{ker}\left(\pi_{z}\right)$ holds. The other inclusion follows from dimensional reasons.

Lemma 3.12. The subspace $\mathfrak{q}_{z}=R\left(\mathfrak{q}_{z}\right) \oplus H\left(\mathfrak{q}_{z}\right)$ is determined by the following.

(i) We have

$$
R\left(\mathfrak{q}_{z}\right)=\tilde{\pi}_{z}^{-1}\left(\mathfrak{g}_{2} \cap \operatorname{Ad}\left(z^{-1}\right) \mathfrak{g}_{1}\right)=\left\{(\operatorname{Ad}(z) \xi,-\xi) ; \xi \in \mathfrak{g}_{2} \cap \operatorname{Ad}\left(z^{-1}\right) \mathfrak{g}_{1}\right\} .
$$

(ii) We have

$$
\left(\widetilde{\pi}_{z}\right)^{-1}\left(\mathfrak{u}_{\lambda, a}^{\mathbb{C}}\right)=\left\{\left(\operatorname{Ad}(z) \sigma_{2}(\xi)+\sigma_{1}\left(\operatorname{Ad}(z) \sigma_{2}(\xi)\right), \xi+\sigma_{2}(\xi)\right) ; \xi \in \mathfrak{u}_{\lambda, a}^{\mathbb{C}}\right\}
$$

for all $(\lambda, a) \in \tilde{\Lambda} \backslash \tilde{\Lambda}(z)$.

Proof. Firstly, we have to show that $\left\{(\operatorname{Ad}(z) \xi,-\xi) ; \xi \in \mathfrak{g}_{2} \cap \operatorname{Ad}\left(z^{-1}\right) \mathfrak{g}_{1}\right\}$ is contained in $\mathfrak{q}_{z}=\mathfrak{g}_{z}^{\perp}$ where the orthogonal complement is taken with respect to the Killing form of $\mathfrak{g}_{1} \oplus \mathfrak{g}_{2}$. Hence, let $\xi, \xi^{\prime} \in \mathfrak{g}_{2} \cap \operatorname{Ad}\left(z^{-1}\right) \mathfrak{g}_{1}$ and consider

$$
\begin{aligned}
B_{\mathfrak{g}_{1} \oplus \mathfrak{g}_{2}}\left((\operatorname{Ad}(z) \xi, \xi),\left(\operatorname{Ad}(z) \xi^{\prime},-\xi^{\prime}\right)\right) & =B_{\mathfrak{g}_{1}}\left(\operatorname{Ad}(z) \xi, \operatorname{Ad}(z) \xi^{\prime}\right)-B_{\mathfrak{g}_{2}}\left(\xi, \xi^{\prime}\right) \\
& =B_{\mathfrak{u}^{\mathbb{C}}}\left(\xi, \xi^{\prime}\right)-B_{\mathfrak{u}^{\mathbb{C}}}\left(\xi, \xi^{\prime}\right)=0 .
\end{aligned}
$$

A simple computation shows $\left\{(\operatorname{Ad}(z) \xi,-\xi) ; \xi \in \mathfrak{g}_{2} \cap \operatorname{Ad}\left(z^{-1}\right) \mathfrak{g}_{1}\right\} \subset R\left(\mathfrak{q}_{z}\right)$. Since the converse inclusion follows from dimensional reasons, the first claim is proven.

A similar argument as above implies that $\left\{\left(\operatorname{Ad}(z) \sigma_{2}(\xi)+\sigma_{1}\left(\operatorname{Ad}(z) \sigma_{2}(\xi)\right), \xi+\right.\right.$ $\left.\left.\sigma_{2}(\xi)\right) ; \xi \in \mathfrak{u}_{\lambda, a}^{\mathbb{C}}\right\}$ lies in $\mathfrak{q}_{z}$. In order to prove the second assertion let $\xi \in \mathfrak{u}_{\lambda, a}^{\mathbb{C}}$ be given and consider

$$
\begin{gathered}
\tilde{\pi}_{z}\left(\operatorname{Ad}(z) \sigma_{2}(\xi)+\sigma_{1}\left(\operatorname{Ad}(z) \sigma_{2}(\xi)\right), \xi+\sigma_{2}(\xi)\right) \\
=\sigma_{2}(\xi)+\operatorname{Ad}\left(z^{-1}\right) \sigma_{1} \operatorname{Ad}(z) \sigma_{2}(\xi)-\xi-\sigma_{2}(\xi) \\
=\tau_{z}(\xi)-\xi=\left(a e^{-2 i \lambda(\eta)}-1\right) \xi=: \varphi_{\lambda, a}(\xi) .
\end{gathered}
$$

Since $\varphi_{\lambda, a}(\xi) \in \mathfrak{u}_{\lambda, a}^{\mathbb{C}}$ holds, the lemma is proven.

Remark 3.13. Note that the map $\varphi_{\lambda, a}: \mathfrak{u}_{\lambda, a}^{\mathbb{C}} \rightarrow \mathfrak{u}_{\lambda, a}^{\mathbb{C}}, \xi \mapsto\left(a e^{-2 i \lambda(\eta)}-1\right) \xi$, is an isomorphism if and only if $(\lambda, a) \notin \widetilde{\Delta}(z)$ holds. In this case the inverse map is given by

$$
\varphi_{\lambda, a}^{-1}(\xi)=\frac{1}{a e^{-2 i \lambda(\eta)}-1} \xi
$$


Definition 3.14. A Levi basis of $H_{z} M_{z}$ is a basis $\left(\xi_{\lambda, a}\right)_{(\lambda, a)}$ of $H_{z} M_{z}$ such that $\xi_{\lambda, a} \in \mathfrak{u}_{\lambda, a}^{\mathbb{C}}$ and $\sigma_{2}\left(\xi_{\lambda, a}\right)=\xi_{\sigma_{2}(\lambda), a}$ hold for all $(\lambda, a) \in \widetilde{\Lambda} \backslash \widetilde{\Lambda}(z)$.

From now on we fix a Levi basis $\left(\xi_{\lambda, a}\right)$ of $H_{z} M_{z}$.

Theorem 3.15. We obtain the following formula for the Levi form of $M_{z}$ :

$$
\mathcal{L}_{z}\left(\xi_{\lambda, a}, \xi_{\mu, b}\right)= \begin{cases}\frac{i}{a e^{-2 i \lambda(\eta)}-1}\left[\xi_{\lambda, a}, \xi_{\sigma_{2}(\mu), b}\right] & \text { if }\left(\lambda+\sigma_{2}(\mu), a b\right) \in \tilde{\Lambda}(z) \\ 0 & \text { else }\end{cases}
$$

Proof. We will start by computing $\tilde{\pi}_{z}^{-1}\left(\xi_{\lambda, a}\right)$ for $\xi_{\lambda, a} \in \mathfrak{u}_{\lambda, a}^{\mathbb{C}}$. Lemma 3.12 gives us

$$
\begin{aligned}
\tilde{\pi}_{z}^{-1}(\xi) & =\left(\operatorname{Ad}(z) \sigma_{2}\left(\varphi_{\lambda, a}^{-1} \xi\right)+\sigma_{1}\left(\operatorname{Ad}(z) \sigma_{2}\left(\varphi_{\lambda, a}^{-1} \xi\right)\right), \varphi_{\lambda, a}^{-1} \xi+\sigma_{2}\left(\varphi_{\lambda, a}^{-1} \xi\right)\right) \\
& =\left(\operatorname{Ad}(z)\left(\sigma_{2}\left(\varphi_{\lambda, a}^{-1} \xi\right)+\tau_{z}\left(\varphi_{\lambda, a}^{-1} \xi\right)\right), \varphi_{\lambda, a}^{-1} \xi+\sigma_{2}\left(\varphi_{\lambda, a}^{-1} \xi\right)\right) \\
& =\left(\operatorname{Ad}(z)\left(\varphi_{\lambda, a}^{-1} \xi+\sigma_{2}\left(\varphi_{\lambda, a}^{-1} \xi\right)\right)+\operatorname{Ad}(z) \xi, \varphi_{\lambda, a}^{-1} \xi+\sigma_{2}\left(\varphi_{\lambda, a}^{-1} \xi\right)\right)
\end{aligned}
$$

for any $\xi \in \mathfrak{u}_{\lambda, a}^{\mathbb{C}}$. In the next step we determine the Lie bracket

$$
\left[\tilde{\pi}_{z}^{-1}\left(\xi_{\lambda, a}\right), \tilde{\pi}_{z}^{-1}\left(\xi_{\mu, b}\right)\right] .
$$

Since the Lie bracket of $\mathfrak{g}=\mathfrak{g}_{1} \oplus \mathfrak{g}_{2}$ is defined component-wise, we consider

$$
\begin{aligned}
& \quad\left[\operatorname{Ad}(z)\left(\varphi_{\lambda, a}^{-1} \xi_{\lambda, a}+\sigma_{2}\left(\varphi_{\lambda, a}^{-1} \xi_{\lambda, a}\right)\right)\right. \\
& \left.\quad+\operatorname{Ad}(z) \xi_{\alpha, \lambda}, \operatorname{Ad}(z)\left(\varphi_{\mu, b}^{-1} \xi_{\mu, b}+\sigma_{2}\left(\varphi_{\mu, b}^{-1} \xi_{\mu, b}\right)\right)+\operatorname{Ad}(z) \xi_{\mu, b}\right] \\
& \quad=\operatorname{Ad}(z)\left[\varphi_{\lambda, a}^{-1} \xi_{\lambda, a}+\sigma_{2}\left(\varphi_{\lambda, a}^{-1} \xi_{\lambda, a}\right)+\xi_{\lambda, a}, \varphi_{\mu, b}^{-1} \xi_{\mu, b}+\sigma_{2}\left(\varphi_{\mu, b}^{-1} \xi_{\mu, b}\right)+\xi_{\mu, b}\right]
\end{aligned}
$$

and

$$
\left[\varphi_{\lambda, a}^{-1} \xi_{\lambda, a}+\sigma_{2}\left(\varphi_{\alpha, \lambda}^{-1} \xi_{\lambda, a}\right), \varphi_{\mu, b}^{-1} \xi_{\mu, b}+\sigma_{2}\left(\varphi_{\mu, b}^{-1} \xi_{\mu, b}\right)\right] .
$$

The application of $\pi_{z}$ to the element in $\mathfrak{g}_{1} \oplus \mathfrak{g}_{2}$ whose components are given by the above gives

$$
\left[\varphi_{\lambda, a}^{-1} \xi_{\lambda, a}+\sigma_{2}\left(\varphi_{\alpha, \lambda}^{-1} \xi_{\lambda, a}\right), \xi_{\mu, b}\right]+\left[\xi_{\lambda, a}, \varphi_{\mu, b}^{-1} \xi_{\mu, b}+\sigma_{2}\left(\varphi_{\mu, b}^{-1} \xi_{\mu, b}\right)\right]+\left[\xi_{\lambda, a}, \xi_{\mu, b}\right] .
$$

By the same computation we obtain for $\pi_{z}\left(\left[\tilde{\pi}_{z}^{-1}\left(\xi_{1}\right), \tilde{\pi}_{z}^{-1}\left(i \xi_{2}\right)\right]\right)$ the following expression:

$\left[\varphi_{\lambda, a}^{-1} \xi_{\lambda, a}+\sigma_{2}\left(\varphi_{\alpha, \lambda}^{-1} \xi_{\lambda, a}\right), i \xi_{\mu, b}\right]+\left[\xi_{\lambda, a}, \varphi_{\mu, b}^{-1} i \xi_{\mu, b}+\sigma_{2}\left(\varphi_{\mu, b}^{-1} i \xi_{\mu, b}\right)\right]+\left[\xi_{\lambda, a}, i \xi_{\mu, b}\right]$. 
To arrive at the Levi form, we have to multiply (3.2) by $\frac{i}{2}$ and subtract (3.3) multiplied by $\frac{1}{2}$. Due to the facts that $\varphi_{\lambda, a}$ and $\varphi_{\mu, b}$ are complex-linear, while $\sigma_{2}$ is anti-linear over $\mathbb{C}$, this leads to

$$
i\left[\xi_{\lambda, a}, \sigma_{2}\left(\varphi_{\mu, b}^{-1} \xi_{\mu, b}\right)\right] .
$$

Inserting the concrete expression for $\varphi_{\mu, b}^{-1}$ yields

$$
\begin{aligned}
& \pi_{z}\left(\frac{i}{2}\left[\tilde{\pi}_{z}^{-1}\left(\xi_{\lambda, a}\right), \tilde{\pi}_{z}^{-1}\left(\xi_{\mu, b}\right)\right]-\frac{1}{2}\left[\tilde{\pi}_{z}^{-1}\left(\xi_{\lambda, a}\right), \tilde{\pi}_{z}^{-1}\left(i \xi_{\mu, b}\right)\right]\right) \\
& =\frac{i}{\mu^{-1} e^{2 i \overline{\beta(\eta)}}-1}\left[\xi_{\lambda, a}, \sigma_{2}\left(\xi_{\mu, b}\right)\right] .
\end{aligned}
$$

To arrive at the Levi form, we have to project this element onto $\left(\mathfrak{g}_{2} \cap \operatorname{Ad}\left(z^{-1}\right) \mathfrak{g}_{1}\right)^{\mathbb{C}}$. Consequently, we only obtain a nonzero contribution if $\left[\xi_{\lambda, a}, \sigma_{2}\left(\xi_{\mu, b}\right)\right] \in \operatorname{Fix}\left(\tau_{z}\right)$ holds. By the definition of a Levi basis this condition translates into the one formulated in the theorem. This finishes the proof.

Remark 3.16. By Theorem 3.15 the nullspace of the Levi form of a generic orbit coincides with

$$
\bigoplus_{(0, a) \in \widetilde{\Lambda}: a \neq 1} \mathfrak{u}_{0, a}^{\mathbb{C}} .
$$

According to [20, Lemma 5.1] the subalgebra $\mathfrak{u}_{0}^{\mathbb{C}}$ is a Cartan subalgebra of $\mathfrak{u}^{\mathbb{C}}$. Since $\operatorname{dim}_{\mathbb{C}} \mathfrak{u}_{0,1}^{\mathbb{C}}=\operatorname{dim}_{\mathbb{C}} \mathfrak{c}^{\mathbb{C}}$ coincides with the rank of $\mathfrak{g}_{1} \cap \mathfrak{g}_{2}$, we conclude that the multiplicity of the eigenvalue 0 of $\mathcal{L}_{z}$ is given by $\operatorname{rk}\left(\mathfrak{u}^{\mathbb{C}}\right)-\operatorname{rk}\left(\mathfrak{g}_{1} \cap \mathfrak{g}_{2}\right)$.

\subsection{The quadratic Levi form}

In this subsection we will derive explicit formulas for the quadratic Levi form of a generic orbit $M_{z}=\left(G_{1} \times G_{2}\right) \cdot z$ from Theorem 3.15. For $(\lambda, a) \in \widetilde{\Lambda}$ we define

$$
\mathfrak{u}^{\mathbb{C}}[\lambda, a]:=\mathfrak{u}_{\lambda, a}^{\mathbb{C}}+\mathfrak{u}_{\sigma_{2}(\lambda), a}^{\mathbb{C}}+\mathfrak{u}_{-\lambda, a^{-1}}^{\mathbb{C}}+\mathfrak{u}_{-\sigma_{2}(\lambda), a^{-1}}^{\mathbb{C}} .
$$

Since the $\mathfrak{u}^{\mathbb{C}}[\lambda, a] \perp \mathfrak{u}^{\mathbb{C}}[\mu, b]$ with respect to the Levi form $\mathcal{L}_{z}$ for $\left(\lambda+\sigma_{2}(\mu), a b\right) \notin$ $\widetilde{\Lambda}(z)=\{(0,1)\}$, the Levi form is determined by its restriction to these spaces for which explicit formulas are given in the next proposition. We make use of the partition $\Lambda \backslash\{0\}=\Lambda_{r} \dot{\cup} \Lambda_{i} \dot{\cup} \Lambda_{c}$.

Proposition 3.17. Let $(\lambda, a) \in \widetilde{\Lambda}$ be given.

(1) For $\lambda \in \Lambda_{r}$ we obtain

$$
\widehat{\mathcal{L}}_{z}\left(r_{\lambda} \xi_{\lambda, a}+r_{-\lambda} \xi_{-\lambda, a^{-1}}\right)=-2 \operatorname{Im}\left(\frac{r_{\lambda} \bar{r}_{-\lambda}}{a e^{-2 i \lambda(\eta)}-1}\right)\left[\xi_{\lambda, a}, \xi_{-\lambda, a^{-1}}\right] .
$$


(2) For $\lambda \in \Lambda_{i}$ and $a=1$ we obtain

$$
\widehat{\mathcal{L}}_{z}\left(r_{\lambda} \xi_{\lambda, 1}+r_{-\lambda} \xi_{-\lambda, 1}\right)=\left(\frac{\left|r_{\lambda}\right|^{2}}{e^{-2 i \lambda(\eta)}-1}-\frac{\left|r_{-\lambda}\right|^{2}}{e^{2 i \lambda(\eta)}-1}\right) i\left[\xi_{\lambda, 1}, \xi_{-\lambda, 1}\right] .
$$

(3) For $\lambda \in \Lambda_{i}$ and $a=-1$ we obtain

$$
\widehat{\mathcal{L}}_{z}\left(r_{\lambda} \xi_{\lambda,-1}+r_{-\lambda} \xi_{-\lambda,-1}\right)=-\left(\frac{\left|r_{\lambda}\right|^{2}}{e^{-2 i \lambda(\eta)}+1}-\frac{\left|r_{-\lambda}\right|^{2}}{e^{2 i \lambda(\eta)}+1}\right) i\left[\xi_{\lambda,-1}, \xi_{-\lambda,-1}\right] .
$$

(4) For $\lambda \in \Lambda_{i}$ and $a \neq \pm 1$ we obtain

$$
\begin{aligned}
& \widehat{\mathcal{L}}_{z}\left(r_{\lambda, a} \xi_{\lambda, a}+r_{\lambda, a^{-1}} \xi_{\lambda, a^{-1}}+r_{-\lambda, a} \xi_{-\lambda, a}+r_{-\lambda, a^{-1}} \xi_{-\lambda, a^{-1}}\right) \\
& =2 \operatorname{Re}\left(\frac{i r_{\lambda, a} \bar{r}_{\lambda, a^{-1}}}{a e^{-2 i \lambda(\eta)}-1}\left[\xi_{\lambda, a}, \xi_{-\lambda, a^{-1}}\right]\right) \\
& \quad+2 \operatorname{Re}\left(\frac{i r_{-\lambda, a} \bar{r}_{-\lambda, a^{-1}}}{a e^{2 i \lambda(\eta)}-1}\left[\xi_{-\lambda, a}, \xi_{\lambda, a^{-1}}\right]\right) .
\end{aligned}
$$

(5) For $\lambda \in \Lambda_{c}$ and $a=1$ we obtain

$$
\begin{aligned}
\widehat{\mathcal{L}}_{z}\left(r_{\lambda} \xi_{\lambda, 1}+s_{\lambda} \sigma_{2}\left(\xi_{\lambda, 1}\right)+r_{-\lambda} \xi_{-\lambda, 1}+s_{-\lambda} \sigma_{2}\left(\xi_{-\lambda, 1}\right)\right) \\
=2 \operatorname{Re}\left(\left(\frac{i r_{\lambda} \bar{s}_{-\lambda}}{e^{-2 i \lambda(\eta)}-1}-\frac{i r_{-\lambda} \bar{s}_{\lambda}}{e^{2 i \lambda(\eta)}-1}\right)\left[\xi_{\lambda, 1}, \xi_{-\lambda, 1}\right]\right) .
\end{aligned}
$$

(6) For $\lambda \in \Lambda_{c}$ and $a=-1$ we obtain

$$
\begin{aligned}
\widehat{\mathcal{L}}_{z}\left(r_{\lambda} \xi_{\lambda,-1}+s_{\lambda} \sigma_{2}\left(\xi_{\lambda,-1}\right)+r_{-\lambda} \xi_{-\lambda,-1}+s_{-\lambda} \sigma_{2}\left(\xi_{-\lambda,-1}\right)\right) \\
=2 \operatorname{Re}\left(\left(\frac{i r_{-\lambda} \bar{s}_{\lambda}}{e^{2 i \lambda(\eta)}+1}-\frac{i r_{\lambda} \bar{s}_{-\lambda}}{e^{-2 i \lambda(\eta)}+1}\right)\left[\xi_{\lambda,-1}, \xi_{-\lambda,-1}\right]\right) .
\end{aligned}
$$

(7) For $\lambda \in \Lambda_{c}$ and $a \neq \pm 1$ we obtain

$$
\begin{aligned}
& \widehat{\mathcal{L}}_{z}\left(r_{\lambda, a} \xi_{\lambda, a}+s_{\lambda, a} \sigma_{2}\left(\xi_{\lambda, a}\right)+r_{-\lambda, a^{-1}} \xi_{-\lambda, a^{-1}}+s_{-\lambda, a^{-1}} \sigma_{2}\left(\xi_{-\lambda, a^{-1}}\right)\right) \\
& =2 \operatorname{Re}\left(\frac{i r_{\lambda, a} \bar{s}_{-\lambda, a^{-1}}}{a e^{-2 i \lambda(\eta)}-1}\left[\xi_{\lambda, a}, \xi_{-\lambda, a^{-1}}\right]\right) \\
& \quad+2 \operatorname{Re}\left(\frac{i r_{-\lambda, a^{-1}} \bar{s}_{\lambda, a}}{a^{-1} e^{2 i \lambda(\eta)}-1}\left[\xi_{-\lambda, a^{-1}}, \xi_{\lambda, a}\right]\right) .
\end{aligned}
$$

Proof. The proof is a straightforward application of Theorem 3.15. As illustration we will prove the first assertion. If $\lambda$ is a real weight, we have $\sigma_{2}(\lambda)=\lambda$ and 
therefore $\mathfrak{u}^{\mathbb{C}}[\lambda, a]=\mathfrak{u}_{\lambda, a}^{\mathbb{C}} \oplus \mathfrak{u}_{-\lambda, a^{-1}}^{\mathbb{C}}$. For arbitrary numbers $r_{\lambda}, r_{-\lambda} \in \mathbb{C}$ we obtain

$$
\begin{aligned}
\widehat{\mathcal{L}}_{z}\left(r_{\lambda} \xi_{\lambda, a}+r_{-\lambda} \xi_{-\lambda, a^{-1}}\right)= & \left|r_{\lambda}\right|^{2} \widehat{\mathcal{L}}_{z}\left(\xi_{\lambda, a}\right)+r_{\lambda} \bar{r}_{-\lambda} \mathcal{L}_{z}\left(\xi_{\lambda, a}, \xi_{-\lambda, a^{-1}}\right) \\
& +\bar{r}_{\lambda} r_{-\lambda} \mathcal{L}_{z}\left(\xi_{-\lambda, a^{-1}}, \xi_{\lambda, a}\right)+\left|r_{-\lambda}\right|^{2} \widehat{\mathcal{L}}_{z}\left(\xi_{-\lambda, a^{-1}}\right) \\
= & 2 \operatorname{Re}\left(r_{\lambda} \bar{r}_{-\lambda} \mathcal{L}_{z}\left(\xi_{\lambda, a}, \xi_{-\lambda, a^{-1}}\right)\right) \\
= & 2 \operatorname{Re}\left(\frac{i r_{\lambda} \bar{r}_{-\lambda}}{a e^{-2 i \lambda(\eta)}-1}\left[\xi_{\lambda, a}, \xi_{-\lambda, a^{-1}}\right]\right) \\
= & 2 \operatorname{Re}\left(\frac{i r_{\lambda} \bar{r}_{-\lambda}}{a e^{-2 i \lambda(\eta)}-1}\right)\left[\xi_{\lambda, a}, \xi_{-\lambda, a^{-1}}\right],
\end{aligned}
$$

since $\sigma_{2}\left[\xi_{\lambda, a}, \xi_{-\lambda, a^{-1}}\right]=\left[\xi_{\lambda, a}, \xi_{-\lambda, a^{-1}}\right]$ for real weights $\lambda$.

\subsection{Reduction to the $\left(\sigma_{1}, \sigma_{2}\right)$-irreducible case}

In this subsection we will introduce the appropriate reduction method in order to facilitate the determination of the Levi cone.

Definition 3.18. We say that $\mathfrak{u}^{\mathbb{C}}$ is $\left(\sigma_{1}, \sigma_{2}\right)$-irreducible if there is no non-trivial ideal in $\mathfrak{u}^{\mathbb{C}}$ which is invariant under $\sigma_{1}$ and $\sigma_{2}$.

The next lemma characterizes $\left(\sigma_{1}, \sigma_{2}\right)$-irreducibility in terms of the set of weights $\Lambda=\Lambda\left(\mathfrak{u}^{\mathbb{C}}, \mathfrak{c}_{0}\right)$ where $\mathfrak{c}_{0}=\mathfrak{t}_{0} \oplus \mathfrak{a}_{0}$ is the fundamental Cartan subalgebra of $\mathfrak{g}_{1} \cap \mathfrak{g}_{2}$.

Lemma 3.19. The Lie algebra $\mathfrak{u}^{\mathbb{C}}$ is $\left(\sigma_{1}, \sigma_{2}\right)$-irreducible if and only if the root system $\Delta:=\Lambda \backslash\{0\} \subset\left(i \mathfrak{t}_{0} \oplus \mathfrak{a}_{0}\right)^{*}$ is irreducible.

Proof. Let us assume that $\mathfrak{u}^{\mathbb{C}}$ is $\left(\sigma_{1}, \sigma_{2}\right)$-irreducible. If the root system $\Delta$ is not irreducible, there is a decomposition $\Delta=\Delta_{1} \cup \dot{\cup} \Delta_{2}$ into non-empty subsystems $\Delta_{1}$, $\Delta_{2}$ such that for all $\lambda_{j} \in \Delta_{j}$ neither of $\lambda_{1} \pm \lambda_{2}$ is a root. It follows that

$$
\mathfrak{u}_{j}^{\mathbb{C}}:=\mathfrak{u}_{0, j}^{\mathbb{C}} \oplus \bigoplus_{\lambda \in \Delta_{j}} \mathfrak{u}_{\lambda}^{\mathbb{C}}
$$

where $\mathfrak{u}_{0, j}^{\mathbb{C}}:=\operatorname{Span}\left\{\left[\mathfrak{u}_{\lambda}^{\mathbb{C}}, \mathfrak{u}_{-\lambda}^{\mathbb{C}}\right] ; \lambda \in \Delta_{j}\right\}$, is a non-trivial ideal invariant under $\sigma_{1}$ and $\sigma_{2}$, which contradicts the fact that $\mathfrak{u}^{\mathbb{C}}$ is $\left(\sigma_{1}, \sigma_{2}\right)$-irreducible.

In order to prove the converse, let us assume that $\mathfrak{u}_{1}^{\mathbb{C}}$ is a non-trivial ideal in $\mathfrak{u}^{\mathbb{C}}$ invariant under $\sigma_{1}$ and $\sigma_{2}$. Consequently, its orthogonal complement $\mathfrak{u}_{2}^{\mathbb{C}}$ with respect to the Killing form $B_{\mathfrak{u}^{\mathbb{C}}}$ is also a non-trivial $\sigma_{1}$ - and $\sigma_{2}$-stable ideal and $\mathfrak{u}^{\mathbb{C}}=\mathfrak{u}_{1}^{\mathbb{C}} \oplus \mathfrak{u}_{2}^{\mathbb{C}}$. It is not hard to see that this decomposition induces similar decompositions of $\mathfrak{g}_{1} \cap \mathfrak{g}_{2}, \mathfrak{c}_{0}$, and hence also of the root system $\Delta$ which contradicts the fact that $\Delta$ is irreducible.

Since the computation of the Levi form is local and the Levi form is invariant under local biholomorphisms, it does no harm to go over to coverings. Hence, we assume that $U^{\mathbb{C}}$ is simply-connected. 
Theorem 3.20. There exists an up to re-ordering unique decomposition $\mathfrak{u}^{\mathbb{C}}=\mathfrak{u}_{1}^{\mathbb{C}} \oplus$ $\cdots \oplus \mathfrak{u}_{N}^{\mathbb{C}}$ into $\left(\sigma_{1}, \sigma_{2}\right)$-irreducible ideals. If $U^{\mathbb{C}}$ is simply-connected, we have the corresponding decomposition of $U^{\mathbb{C}}$, of the real forms $G_{1}$ and $G_{2}$, and of the orbits and their (complex) tangent spaces. This decomposition of the complex tangent space of a closed orbit is orthogonal with respect to its Levi form. Consequently, the Levi cone is the direct product of the Levi cones of each factor.

Proof. Since $\mathfrak{u}^{\mathbb{C}}$ is semi-simple, it is the direct sum of its simple ideals, and each of these is $\theta$-invariant. Since $\sigma_{1}$ and $\sigma_{2}$ are automorphisms of $\mathfrak{u}^{\mathbb{C}}$, they map simple ideals onto simple ideals. This observation proves that $\mathfrak{u}^{\mathbb{C}}$ has a unique decomposition into $\left(\sigma_{1}, \sigma_{2}\right)$-irreducible ideals. Moreover, the simple ideals which appear in one $\left(\sigma_{1}, \sigma_{2}\right)$-irreducible ideal must be all isomorphic.

Let $\mathfrak{u}^{\mathbb{C}}=\mathfrak{u}_{1}^{\mathbb{C}} \oplus \cdots \oplus \mathfrak{u}_{N}^{\mathbb{C}}$ denote this decomposition and let $U_{k}^{\mathbb{C}}$ be the subgroup of $U^{\mathbb{C}}$ with Lie algebra $\mathfrak{u}_{k}^{\mathbb{C}}$. Since $U^{\mathbb{C}}$ is simply-connected, we obtain

$$
U^{\mathbb{C}} \cong U_{1}^{\mathbb{C}} \times \cdots \times U_{N}^{\mathbb{C}},
$$

and since each semi-simple normal subgroup $U_{k}^{\mathbb{C}}$ is invariant under $\sigma_{1}$ and $\sigma_{2}$, we have similar decompositions

$$
G_{j} \cong\left(G_{j}\right)_{1} \times \cdots \times\left(G_{j}\right)_{N}
$$

for $j=1,2$. Here, $\left(G_{j}\right)_{k}$ is the fixed point set of $\left.\sigma_{j}\right|_{U_{k}^{\mathbb{C}}}$. It follows that the $\left(G_{1} \times G_{2}\right)$-orbits are also direct products of their intersections with the normal subgroups $U_{k}^{\mathbb{C}}$. Since the $\mathfrak{u}_{k}^{\mathbb{C}}$ are ideals, we have a corresponding decomposition of the set of weights into strongly orthogonal subsystems. Finally, the computation of the Levi form in Theorem 3.15 tells us that the respective parts of the complex tangent spaces are Levi-orthogonal.

\subsection{The Levi cone}

In this subsection we will determine the full Levi cone of a generic $\left(G_{1} \times G_{2}\right)$-orbit. We assume that $U^{\mathbb{C}}$ is simply-connected and that $\mathfrak{u}^{\mathbb{C}}$ is $\left(\sigma_{1}, \sigma_{2}\right)$-irreducible.

Let $z=n \exp (i \eta)$ be a regular element contained in the standard Cartan subset $C:=n \exp (i \mathrm{c})$. Since $z$ is regular, we have $e^{2 i \lambda(\eta)} \neq 1$ for all $(\lambda, 1) \in \widetilde{\Lambda}$. Hence, we conclude $\lambda(\eta) \neq 0$ for all $(\lambda, 1) \in \widetilde{\Lambda}_{i}$. The following lemma is then a direct consequence of Proposition 3.17.

Lemma 3.21. The Levi cone $\mathcal{C}_{z}$ of the generic orbit $\left(G_{1} \times G_{2}\right) \cdot z$ is generated by

(1) $\pm\left[\xi_{\lambda, a}, \xi_{-\lambda, a^{-1}}\right]$ for $(\lambda, a) \in \widetilde{\Lambda}_{r}$,

(2) $-i\left[\xi_{\lambda, 1}, \xi_{-\lambda, 1}\right]$ for $(\lambda, 1) \in \widetilde{\Lambda}_{i}$ with $\lambda(\eta)>0$,

(3) $i\left[\xi_{\lambda, 1}, \xi_{-\lambda, 1}\right]$ for $(\lambda, 1) \in \widetilde{\Lambda}_{i}$ with $\lambda(\eta)<0$,

(4) $\pm i\left[\xi_{\lambda,-1}, \xi_{-\lambda,-1}\right]$ for $(\lambda,-1) \in \widetilde{\Lambda}_{i}$,

(5) $\pm \operatorname{Re}\left(\left[\xi_{\lambda, a}, \xi_{-\lambda, a^{-1}}\right]\right)$ and $\pm \operatorname{Im}\left(\left[\xi_{\lambda, a}, \xi_{-\lambda, a^{-1}}\right]\right)$ for $(\lambda, a) \in \tilde{\Lambda}_{i}$ with $\lambda \neq \pm 1$, and 
(6) $\pm \operatorname{Re}\left(\left[\xi_{\lambda, a}, \xi_{-\lambda, a^{-1}}\right]\right)$ and $\pm \operatorname{Im}\left(\left[\xi_{\lambda, a}, \xi_{-\lambda, a^{-1}}\right]\right)$ for $(\lambda, a) \in \widetilde{\Lambda}_{c}$.

Remark 3.22. Since we have defined the real structure on $\mathfrak{c}^{\mathbb{C}}$ via $\sigma_{2}$, we obtain

$$
\begin{aligned}
\operatorname{Re}\left(\left[\xi_{\lambda, a}, \xi_{-\lambda, a^{-1}}\right]\right) & =\left[\xi_{\lambda, \lambda}, \xi_{-\lambda, a^{-1}}\right]+\sigma_{2}\left(\left[\xi_{\lambda, a}, \xi_{-\lambda, a^{-1}}\right]\right) \\
& =\left[\xi_{\lambda, a}, \xi_{-\lambda, a^{-1}}\right]+\left[\xi_{\sigma_{2}(\lambda), \lambda}, \xi_{-\sigma_{2}(\lambda), \lambda}\right] .
\end{aligned}
$$

The imaginary part $\operatorname{Im}\left(\left[\xi_{\lambda, a}, \xi_{-\lambda, a^{-1}}\right]\right)$ can be expressed by an analogous formula.

In order to state the main theorem we have to review some properties of real simple Lie algebras of Hermitian type. For a more detailed exposition of these topics we refer the reader to [12] and [24].

Recall that a simple real Lie algebra $\mathfrak{g}=\mathfrak{k} \oplus \mathfrak{p}$ is said to be of Hermitian type if the center of $\mathfrak{k}$ is non-trivial. This condition implies that a maximal torus $\mathfrak{t} \subset \mathfrak{k}$ is a Cartan subalgebra of $\mathfrak{g}$. Then every root $\alpha$ in $\Delta=\Delta\left(\mathfrak{g}^{\mathbb{C}}, \mathfrak{t}\right)$ is imaginary, and either $\mathfrak{g}_{\alpha}^{\mathbb{C}} \subset \mathfrak{k}^{\mathbb{C}}$ or $\mathfrak{g}_{\alpha}^{\mathbb{C}} \subset \mathfrak{p}^{\mathbb{C}}$ holds. In the first case we call $\alpha$ a compact root, while in the second case $\alpha$ is said to be non-compact. We write $\Delta_{\mathfrak{k}}$ and $\Delta_{\mathfrak{p}}$ for the sets of compact and non-compact roots, respectively. Since $\mathfrak{g}$ is Hermitian, the root system $\Delta$ possesses a good ordering, i.e. there is a choice of the set $\Delta^{+}$of positive roots such that each positive non-compact root is larger than every compact root. This is equivalent to the fact that the set $\Delta_{\mathfrak{p}}^{+}$is invariant under the Weyl group $W\left(\Delta_{\mathfrak{k}}\right)$. There are two natural $W\left(\Delta_{\mathfrak{k}}\right)$-invariant cones $C_{\min } \subset C_{\max }$, where $C_{\min }$ is the closed convex cone generated by

$$
\left\{-i\left[\xi_{\alpha}, \sigma\left(\xi_{\alpha}\right)\right] ; \xi_{\alpha} \in \mathfrak{g}_{\alpha}^{\mathbb{C}}, \alpha \in \Delta_{\mathfrak{p}}^{+}\right\} \subset \mathfrak{t}
$$

and

$$
C_{\max }:=\left\{\eta \in \mathfrak{t} ; i \alpha(\eta) \geq 0 \text { for all } \alpha \in \Delta_{\mathfrak{p}}^{+}\right\} .
$$

Let $C_{\max }^{0}$ be the interior of $C_{\max }$. Then the open subset $G \exp \left(i C_{\max }^{0}\right) G \subset G^{\mathbb{C}}$ is closed under multiplication and hence a semi-group, called the open complex Ol'shanskiĭ semi-group.

Theorem 3.23. Let $\mathfrak{u}^{\mathbb{C}}$ be $\left(\sigma_{1}, \sigma_{2}\right)$-irreducible and let $\left(G_{1} \times G_{2}\right) \cdot z$ be a generic orbit where $z=n \exp (i \eta)$ lies in the standard Cartan subset $C:=n \exp (i \mathfrak{c})$.

(1) If the standard Cartan subspace $\mathfrak{c}$ is non-compact, then $\mathcal{C}_{z}=\mathfrak{c}$ holds.

(2) If $\mathfrak{c}$ is compact and if $a \neq 1$ for some $(\lambda, a) \in \widetilde{\Lambda}$, then we have $\mathcal{C}_{z}=\mathfrak{c}$.

(3) If $\mathfrak{c}$ is compact and if $a=1$ for all generalized weights, then $\sigma_{1}=\sigma_{2}$ holds and there are the following cases.

(i) If $\mathfrak{g}_{1}=\mathfrak{g}_{2}=: \mathfrak{g}$ is of Hermitian type and if $\eta$ lies in $C_{\max }$, then the Levi cone $\mathcal{C}_{z}$ is isomorphic to the dual of the positive Weyl chamber defined by $\Lambda^{+}$. In particular, the Levi cone is pointed.

(ii) If $\mathfrak{g}$ is of Hermitian type and $\eta \notin C_{\max }$, then $\mathcal{C}_{z}=\mathfrak{c}$.

(iii) If $\mathfrak{g}$ is not of Hermitian type, then $\mathcal{C}_{z}=\mathfrak{c}$. 
Remark 3.24. The reader will note that the statement of Theorem 3.23 differs also for the case $\sigma_{1}=\sigma_{2}$ from the corresponding [10, Theorem 5.3]. Indeed, as L. Geatti has kindly pointed out, the formulation of the third part of [10, Theorem 5.3] is not correct. The correct statement in Theorem 3.23 and its proof in the case $\sigma_{1}=\sigma_{2}$ are due to an unpublished erratum written by L. Geatti.

It will turn out to be convenient to express the generators of the Levi cone in terms of the coroots $\eta_{\lambda} \in i \mathfrak{t} \oplus \mathfrak{a}$. Therefore we will identify $\mathfrak{c}=\mathfrak{t} \oplus \mathfrak{a}$ with $i \mathfrak{t} \oplus \mathfrak{a}$ via the map $\left(\eta_{1}, \eta_{2}\right) \mapsto\left(i \eta_{1}, \eta_{2}\right)$. By abuse of notation, we denote the image of the Levi cone under this map again by $\mathcal{C}_{z} \subset i \mathfrak{t} \oplus \mathfrak{a}$. According to Lemma 2.13 we have

$$
\left[\xi_{\lambda, a}, \xi_{-\lambda, a^{-1}}\right]=B_{\mathfrak{u}^{\mathbb{C}}}\left(\xi_{\lambda, a}, \xi_{-\lambda, a^{-1}}\right) \eta_{\lambda} \in \mathbb{C} \eta_{\lambda}
$$

Hence, we can normalize the $\xi_{\lambda, a}$ such that $\left[\xi_{\lambda, a}, \xi_{-\lambda, a^{-1}}\right]=\eta_{\lambda}$ holds for all $\lambda \in$ $\Lambda^{+} \backslash \Lambda_{i}$ and $\left[\xi_{\lambda, a}, \xi_{-\lambda, a^{-1}}\right]= \pm \eta_{\lambda}$ holds for $\lambda \in \Lambda_{i}^{+}$depending on the sign of $B_{\mathfrak{u}^{\mathbb{C}}}\left(\xi_{\lambda, a}, \xi_{-\lambda, a^{-1}}\right)$.

Remark 3.25. In the case where $\mathfrak{g}_{1}=\mathfrak{g}_{2}=$ : $\mathfrak{g}$ and $\mathfrak{t}$ is a compact Cartan subalgebra of $\mathfrak{g}$, we obtain after the above normalization

$$
\left[\xi_{\alpha}, \xi_{-\alpha}\right]= \begin{cases}\eta_{\alpha} & \text { for } \alpha \in \Delta_{\mathfrak{p}}^{+} \\ -\eta_{\alpha} & \text { for } \alpha \in \Delta_{\mathfrak{k}}^{+}\end{cases}
$$

since the real part of $B_{\mathfrak{u}}$ is positive definite on $\mathfrak{p}$ and negative definite on $\mathfrak{k}$.

Proof of Theorem 3.23. (1) Let $\mathfrak{c}$ be non-compact. Since $\Lambda \backslash\{0\}$ satisfies the axioms for an abstract root system, we may choose a set $\Pi \subset \Lambda^{+}$of simple weights. By Lemma 3.21 we know that $\pm \eta_{\lambda}$ lies in $\mathcal{C}_{z}$ for $\lambda \in \Lambda \backslash \Lambda_{i}$, and we have to show that $\pm \eta_{\lambda} \in \mathcal{C}_{z}$ holds for all $\lambda \in \Lambda$. It is enough to prove this fact for all $\eta_{\lambda}$ with $\lambda \in \Pi_{i}:=\Pi \cap \Lambda_{i}$.

If $\lambda, \mu \in \Pi_{i}$ with $\lambda+\mu \in \Lambda$ are given, then $\lambda+\mu \in \Lambda_{i}^{+}$holds. Since $\mathfrak{c}$ is non-compact, this observation implies that there exists an element $\mu \in \Pi \backslash \Pi_{i}$. Let $\lambda \in \Pi_{i}$ be arbitrary (if $\Pi_{i}=\emptyset$, the proof is finished). Since $\Lambda \backslash\{0\}$ is irreducible by Lemma 3.19, its Dynkin diagram is connected and hence we find a sequence $\lambda=\lambda_{1}, \ldots, \lambda_{N}=\mu$ of simple roots which are adjacent in the Dynkin diagram. Consequently, we obtain $\lambda_{j}+\cdots+\lambda_{N} \in \Lambda \backslash \Lambda_{i}$ for all $0 \leq j \leq N-1$. This implies

$$
\pm \eta_{\lambda_{j}+\cdots+\lambda_{N}}= \pm\left(\eta_{\lambda_{j}+\cdots+\lambda_{N-1}}+\eta_{\lambda_{N}}\right) \in \mathcal{C}_{z}
$$

for all $0 \leq j \leq N-1$. Since $\pm \eta_{\lambda_{N}}$ lies in $\mathcal{C}_{z}$, we conclude $\pm \eta_{\lambda_{j}+\cdots+\lambda_{N-1}} \in \mathcal{C}_{z}$ for all $j$. Iterating this argument we finally arrive at $\pm \eta_{\lambda} \in \mathcal{C}_{z}$ which was to be shown.

(2) Let us assume that $\mathfrak{c}$ is compact and that there exists $(\lambda, a) \in \widetilde{\Lambda}$ with $a \neq 1$. In this case we have $\Lambda=\Lambda_{i}$ and $\pm \eta_{\lambda} \in \mathcal{C}_{z}$ for all $\lambda$ such that there exists $a \neq 1$ with $(\lambda, a) \in \widetilde{\Lambda}$. If there are two weights $\lambda_{1}, \lambda_{2} \in \Lambda$ such that $\left(\lambda_{j}, a\right) \in \widetilde{\Lambda}$ implies $a=1$ for $j=1,2$ and such that $\lambda_{1}+\lambda_{2}$ is again a weight, then we conclude from Proposition 2.14 that

$$
\left(\lambda_{1}+\lambda_{2}, a\right) \in \widetilde{\Lambda} \Longrightarrow a=1
$$


holds. Consequently, each set $\Pi \subset \Lambda^{+}$of simple roots must contain a root $\mu$ with $\pm \eta_{\mu} \in \mathcal{C}_{z}$. Now the claim follows from the same argument as above.

(3) Let $\mathfrak{c}=\mathfrak{t}$ be a compact Cartan subalgebra of $\mathfrak{g}_{2} \cap \operatorname{Ad}\left(n^{-1}\right) \mathfrak{g}_{1}$ such that $a=1$ holds for each extended weight $(\lambda, a) \in \widetilde{\Lambda}$. It is enough to prove that this assumption implies $\mathfrak{g}_{1}=\mathfrak{g}_{2}$ since then the claim follows from [10] and Geatti's erratum.

The proof of $\mathfrak{g}_{1}=\mathfrak{g}_{2}$ relies on the comparison of the weight space decompositions

$$
\mathfrak{u}^{\mathbb{C}}=\mathfrak{t}^{\mathbb{C}} \oplus \bigoplus_{\lambda \in \Lambda \backslash\{0\}} \mathfrak{u}_{\lambda, 1}^{\mathbb{C}} \quad \text { and } \quad\left(\mathfrak{g}_{2} \cap \operatorname{Ad}\left(n^{-1}\right) \mathfrak{g}_{1}\right)^{\mathbb{C}}=\mathfrak{t}^{\mathbb{C}} \oplus \bigoplus_{\lambda \in \Lambda^{\prime}} \mathfrak{u}_{\lambda, 1}^{\mathbb{C}},
$$

where $\Lambda^{\prime}$ denotes the set of non-zero weights $\lambda$ for which $\mathfrak{u}_{\lambda, 1}^{\mathbb{C}}$ is contained in $\left(\mathfrak{g}_{2} \cap \operatorname{Ad}\left(n^{-1}\right) \mathfrak{g}_{1}\right)^{\mathbb{C}}$. Note that this is well-defined since $\operatorname{dim} \mathfrak{u}_{\lambda, 1}^{\mathbb{C}}=1$ by Proposition 2.14. Since the weight space decomposition is in both cases defined with respect to $\mathfrak{t}$, a basis of $\Lambda^{\prime}$ has to be a basis of $\Lambda \backslash\{0\}$, too. Since the root system $\Lambda \backslash\{0\}$ is reduced by Proposition 2.14, we conclude

$$
\mathfrak{u}^{\mathbb{C}}=\left(\mathfrak{g}_{2} \cap \operatorname{Ad}\left(n^{-1}\right) \mathfrak{g}_{1}\right)^{\mathbb{C}},
$$

and hence, that $\mathfrak{g}_{2} \cap \operatorname{Ad}\left(n^{-1}\right) \mathfrak{g}_{1}$ is a real form of $\mathfrak{u}^{\mathbb{C}}$. For dimensional reasons this implies

$$
\mathfrak{g}_{2}=\mathfrak{g}_{2} \cap \operatorname{Ad}\left(n^{-1}\right) \mathfrak{g}_{1}=\operatorname{Ad}\left(n^{-1}\right) \mathfrak{g}_{1},
$$

i.e. $\sigma_{2}=\operatorname{Ad}\left(n^{-1}\right) \sigma_{1} \operatorname{Ad}(n)$.

By the definition of a standard Cartan subset, the fundamental Cartan subalgebra $\mathfrak{c}_{0} \subset \mathfrak{g}_{1} \cap \mathfrak{g}_{2}$ has the same dimension as $\mathfrak{t}$. Therefore, we obtain

$$
\operatorname{rk}\left(\mathfrak{g}_{1} \cap \mathfrak{g}_{2}\right)=\operatorname{dim} \mathfrak{c}_{0}=\operatorname{dim} \mathfrak{t}=\operatorname{rk}\left(\mathfrak{g}_{2} \cap \operatorname{Ad}\left(n^{-1}\right) \mathfrak{g}_{1}\right)^{\mathbb{C}}=\operatorname{rk} \mathfrak{u}^{\mathbb{C}},
$$

which implies in the same way as above that $\mathfrak{g}_{1} \cap \mathfrak{g}_{2}$ is a real form of $\mathfrak{u}^{\mathbb{C}}$ and hence that $\mathfrak{g}_{1}=\mathfrak{g}_{2}$ holds.

\section{Applications}

\subsection{The criterion of Fels and Geatti}

We restate Corollary 5.6 from [10] whose proof relies on Theorem 3.4.

Theorem 4.1 (Fels, Geatti). Let $Z$ be a complex manifold on which the Lie group $G$ acts by holomorphic transformations. Let the orbit $M_{z}=G \cdot z$ be a generic CR submanifold such that $\mathcal{C}_{z}=T_{z} M_{z} / H_{z} M_{z}$ holds. Then there exists no $G$-invariant Stein domain in $Z$ which contains $M_{z}$ in its boundary. Furthermore, there is no nonconstant G-invariant plurisubharmonic function which is defined in a neighborhood of $M_{z}$. 
Theorem 4.1 gives a necessary condition for an invariant domain with a generic orbit in its boundary to be Stein. In our situation we obtain the following result.

Theorem 4.2. Let $C=n \exp (i \mathrm{c})$ be a standard Cartan subset and let $\Omega$ be a connected component of the open set $G_{1}\left(C \cap U_{s r}^{\mathbb{C}}\right) G_{2}$. Then $\Omega$ does not contain any proper $\left(G_{1} \times G_{2}\right)$-invariant Stein subdomain unless $\mathfrak{c}$ is compact and $\tau_{n}$ has $a=1$ as only eigenvalue. In this case $G_{1}=G_{2}$ must be of Hermitian type and $\Omega$ is a translate of the open Ol'shanskĭ semi-group in $U^{\mathbb{C}}$.

Consequently, we see that in the case $G_{1} \neq G_{2}$ there are no invariant Stein subdomains in $U^{\mathbb{C}}$ in whose boundary a generic orbit lies. The reader should note that there are only finitely many $\left(G_{1} \times G_{2}\right)$-invariant domains whose boundaries consist entirely of non-generic orbits.

\section{2. $q$-pseudo-convex functions and $q$-completeness}

In this subsection we quickly review the notions of $q$-pseudo-convex functions and $q$-complete complex manifolds. Let $\Omega$ be a domain in a complex manifold $Z$. We call a smooth function on $\Omega$ strictly $q$-pseudo-convex if its Levi form has at least $n-q$ positive eigenvalues, $n:=\operatorname{dim}_{\mathbb{C}} \Omega$, at each point of $\Omega$. Hence, a strictly 0 -pseudo-convex function is the same as a strictly plurisubharmonic function. If $\Omega$ admits a strictly $q$-pseudo-convex exhaustion function, we say that $\Omega$ is $q$-complete. The solution of the Levi problem implies that a domain is Stein if and only if it is 0 -complete. For more properties of $q$-complete complex spaces we refer the reader to [1].

Remark 4.3. A standard argument of complex analysis (compare [24, Corollary XIII.5.4] for the case $q=0$ and [7] for the generalization to $q>0$ ) shows that a domain $\Omega$ in a Stein manifold $Z$ is $q$-complete if and only if there exists a nonnegative strictly $q$-pseudo-convex function $\Phi$ on $\Omega$ with the property $\Phi\left(z_{n}\right) \rightarrow \infty$ whenever $z_{n} \rightarrow z \in \partial \Omega$.

A domain $\Omega \subset Z$ with smooth boundary is called Levi- $q$-convex, if the boundary $\partial \Omega$ can locally be defined by a function whose Levi form has at most $q$ negative eigenvalues when restricted to the complex tangent space at any point of $\partial \Omega$.

By a theorem of Docquier and Grauert [8] a domain $\Omega$ with smooth boundary in a Stein manifold $Z$ is Stein if and only if it is Levi-0-convex. In [9] this result is generalized to arbitrary $q$.

Theorem 4.4 (Oka, Docquier-Grauert, Eastwood-Suria). Let Z be a Stein manifold and let $\Omega \subset Z$ be a domain with smooth boundary. Then $\Omega$ is strictly $q$ complete if and only if $\Omega$ is Levi-q-convex.

\subsection{The rank one case}

If the closed orbit $M_{z}=\left(G_{1} \times G_{2}\right) \cdot z$ is a hypersurface in $U^{\mathbb{C}}$, its intrinsic Levi form coincides with the classical Levi form of that hypersurface, and hence the signature 
of $\widehat{\mathcal{L}}_{z}$ is defined. According to Theorem 4.4 this signature encodes information about complex-analytic properties of the domains bounded by $M_{z}$. In this subsection we will use Matsuki's classification of pairs of involutive automorphisms of simply-connected compact Lie groups in order to classify the triples $\left(U^{\mathbb{C}}, G_{1}, G_{2}\right)$ where $U^{\mathbb{C}}$ is simply-connected and the generic $\left(G_{1} \times G_{2}\right)$-orbit is a hypersurface. Moreover, we will determine the signature of the Levi form of each generic hypersurface orbit.

In [20] pairs of involutive automorphisms of simply-connected semi-simple compact Lie groups are classified under the following notion of equivalence.

Definition 4.5. Let $U$ be a simply-connected semi-simple compact Lie group. Two pairs of involutive automorphisms $\left(\sigma_{1}, \sigma_{2}\right)$ and $\left(\sigma_{1}^{\prime}, \sigma_{2}^{\prime}\right)$ are called equivalent if there exist an automorphism $\varphi \in \operatorname{Aut}(U)$ and an element $u \in U$ such that

$$
\sigma_{1}^{\prime}=\varphi \sigma_{1} \varphi^{-1} \quad \text { and } \quad \sigma_{2}^{\prime}=\operatorname{Int}(u) \varphi \sigma_{2} \varphi^{-1} \operatorname{Int}(u)^{-1}
$$

hold.

Since in our case the involutive automorphisms $\sigma_{1}, \sigma_{2}: U^{\mathbb{C}} \rightarrow U^{\mathbb{C}}$ commute with $\theta$ and are anti-holomorphic, they are completely determined by their restrictions to $U$. Therefore, we may apply the classification result from [20].

Theorem 4.6 (Matsuki). Let $U^{\mathbb{C}}$ be simply-connected. If the generic $\left(G_{1} \times G_{2}\right)$ orbit is a hypersurface in $U^{\mathbb{C}}$, then $U^{\mathbb{C}}$ is of the form

$$
U^{\mathbb{C}}=\underbrace{S \times \cdots \times S}_{k \text { times }}
$$

where $S$ is a $\theta$-stable normal subgroup of $U^{\mathbb{C}}$ either isomorphic to $\operatorname{SL}(2, \mathbb{C})$ or $\operatorname{SL}(3, \mathbb{C})$. Let $\sigma$ and $\tau$ be anti-holomorphic involutive automorphisms of $S$ commuting with $\left.\theta\right|_{S}$. If $k$ is odd, then we have

$$
\begin{aligned}
& \sigma_{1}\left(g_{1}, \ldots, g_{k}\right)=\left(\sigma\left(g_{1}\right), \theta\left(g_{3}\right), \theta\left(g_{2}\right), \ldots, \theta\left(g_{k}\right), \theta\left(g_{k-1}\right)\right) \\
& \sigma_{2}\left(g_{1}, \ldots, g_{k}\right)=\left(\theta\left(g_{2}\right), \theta\left(g_{1}\right), \ldots, \theta\left(g_{k-1}\right), \theta\left(g_{k-2}\right), \tau\left(g_{k}\right)\right),
\end{aligned}
$$

and if $k$ is even, then

$$
\begin{aligned}
& \sigma_{1}\left(g_{1}, \ldots, g_{k}\right)=\left(\sigma\left(g_{1}\right), \theta\left(g_{3}\right), \theta\left(g_{2}\right), \ldots, \theta\left(g_{k-1}\right), \theta\left(g_{k-2}\right), \tau\left(g_{k}\right)\right) \\
& \sigma_{2}\left(g_{1}, \ldots, g_{k}\right)=\left(\theta\left(g_{2}\right), \theta\left(g_{1}\right), \ldots, \theta\left(g_{k}\right), \theta\left(g_{k-1}\right)\right)
\end{aligned}
$$

holds. If $S=\operatorname{SL}(2, \mathbb{C})$, then the pair $(\sigma, \tau)$ is equivalent to one of $\left\{\left(\sigma_{1,1}, \sigma_{1,1}\right)\right.$, $\left.\left(\sigma_{1,1}, \theta\right),(\theta, \theta)\right\}$, where $\sigma_{1,1}$ is the involution defining the non-compact real form $\mathrm{SU}(1,1)$ of $\mathrm{SL}(2, \mathbb{C})$. If $S=\mathrm{SL}(3, \mathbb{C})$, then the only possibility for $(\sigma, \tau)$ up to equivalence is $(\sigma(g), \tau(g))=\left(\bar{g}, I_{2,1} \theta(g) I_{2,1}\right)$ with $I_{2,1}:=\left(\begin{array}{ccc}1 & 0 & 0 \\ 0 & 1 & 0 \\ 0 & 0 & -1\end{array}\right)$. 
Proof. Since the semi-simple complex Lie group $U^{\mathbb{C}}$ is assumed to be simplyconnected, we can identify the automorphism group $\operatorname{Aut}\left(U^{\mathbb{C}}\right)$ with $\operatorname{Aut}\left(\mathfrak{u}^{\mathbb{C}}\right)$. By [20, Proposition 2.2] there exists a $\theta$-invariant decomposition

$$
\mathfrak{u}^{\mathbb{C}}=\mathfrak{u}_{1}^{\mathbb{C}} \oplus \cdots \oplus \mathfrak{u}_{N}^{\mathbb{C}}
$$

into $\sigma_{1}$ - and $\sigma_{2}$-invariant semi-simple ideals $\mathfrak{u}_{j}^{\mathbb{C}}$. Moreover, each $\mathfrak{u}_{j}^{\mathbb{C}}$ is of the form

$$
\mathfrak{u}_{j}^{\mathbb{C}}=\underbrace{\mathfrak{s}_{j} \oplus \cdots \oplus \mathfrak{s}_{j}}_{k_{j} \text { times }},
$$

where $\mathfrak{s}_{j}$ is a $\theta$-stable simple ideal in $\mathfrak{u}_{j}^{\mathbb{C}}$, such that the restriction of the pair $\left(\sigma_{1}, \sigma_{2}\right)$ (or $\left.\left(\sigma_{2}, \sigma_{1}\right)\right)$ to $\mathfrak{u}_{j}^{\mathbb{C}}$ is equivalent to one of the following three types:

(1) The number $k_{j}$ is even and

$$
\begin{aligned}
& \sigma_{1}\left(\xi_{1}, \ldots, \xi_{k}\right)=\left(\varphi\left(\xi_{k}\right), \theta\left(\xi_{3}\right), \theta\left(\xi_{2}\right), \ldots, \theta\left(\xi_{k-1}\right), \theta\left(\xi_{k-2}\right), \varphi^{-1}\left(\xi_{1}\right)\right) \\
& \sigma_{2}\left(\xi_{1}, \ldots, \xi_{k}\right)=\left(\theta\left(\xi_{2}\right), \theta\left(\xi_{1}\right), \ldots, \theta\left(\xi_{k}\right), \theta\left(\xi_{k-1}\right)\right),
\end{aligned}
$$

for some $\mathbb{C}$-anti-linear automorphism $\varphi$ of $\mathfrak{s}_{j}$ commuting with $\left.\theta\right|_{\mathfrak{s}_{j}}$.

(2) The number $k$ is even and

$$
\begin{aligned}
& \sigma_{1}\left(\xi_{1}, \ldots, \xi_{k}\right)=\left(\sigma\left(\xi_{1}\right), \theta\left(\xi_{3}\right), \theta\left(\xi_{2}\right), \ldots, \theta\left(\xi_{k-1}\right), \theta\left(\xi_{k-2}\right), \tau\left(\xi_{k}\right)\right) \\
& \sigma_{2}\left(\xi_{1}, \ldots, \xi_{k}\right)=\left(\theta\left(\xi_{2}\right), \theta\left(\xi_{1}\right), \ldots, \theta\left(\xi_{k}\right), \theta\left(\xi_{k-1}\right)\right),
\end{aligned}
$$

where $\sigma$ and $\tau$ are $\mathbb{C}$-anti-linear involutive automorphisms of $\mathfrak{s}_{j}$ commuting with $\left.\theta\right|_{\mathfrak{s}_{j}}$.

(3) The number $k$ is odd and

$$
\begin{aligned}
& \sigma_{1}\left(\xi_{1}, \ldots, \xi_{k}\right)=\left(\sigma\left(\xi_{1}\right), \theta\left(\xi_{3}\right), \theta\left(\xi_{2}\right), \ldots, \theta\left(\xi_{k}\right), \theta\left(\xi_{k-1}\right)\right) \\
& \sigma_{2}\left(\xi_{1}, \ldots, \xi_{k}\right)=\left(\theta\left(\xi_{2}\right), \theta\left(\xi_{1}\right), \ldots, \theta\left(\xi_{k-1}\right), \theta\left(\xi_{k-2}\right), \tau\left(\xi_{k}\right)\right),
\end{aligned}
$$

where $\sigma$ and $\tau$ are $\mathbb{C}$-anti-linear involutive automorphisms of $\mathfrak{s}_{j}$ commuting with $\left.\theta\right|_{\mathfrak{s}_{j}}$.

The condition that the generic $\left(G_{1} \times G_{2}\right)$-orbit is a hypersurface is equivalent to $\operatorname{rk}\left(\mathfrak{g}_{1} \cap \mathfrak{g}_{2}\right)=1$. In particular, this condition implies that $\mathfrak{u}^{\mathbb{C}}$ is $\left(\sigma_{1}, \sigma_{2}\right)$-irreducible, and we have $\mathfrak{u}^{\mathbb{C}}=\mathfrak{s} \oplus \cdots \oplus \mathfrak{s}(k$ times) for a simple complex Lie algebra $\mathfrak{s}$.

If $\mathfrak{u}^{\mathbb{C}}$ is of the first type, one checks directly that

$$
\mathfrak{g}_{1} \cap \mathfrak{g}_{2} \cong\left(\mathfrak{s}^{\theta \varphi}\right)^{\mathbb{R}}
$$

holds. Consequently $\operatorname{rk}\left(\mathfrak{g}_{1} \cap \mathfrak{g}_{2}\right)$ is even and in particular larger than 1 . This excludes the first type. 
Let $\mathfrak{u}^{\mathbb{C}}$ be of the second or the third type. Again it is not hard to see that

$$
\mathfrak{g}_{1} \cap \mathfrak{g}_{2} \cong \mathfrak{s}^{\sigma} \cap \mathfrak{s}^{\tau}
$$

holds. It follows that the simple complex Lie algebra $\mathfrak{s}$ contains the complex subalgebra $\left(\mathfrak{g}_{1} \cap \mathfrak{g}_{2}\right)^{\mathbb{C}}$ which is given as the set of fixed points of the $\mathbb{C}$-linear semisimple automorphism $\sigma \tau$. Hence, we have to find all pairs $(\mathfrak{s}, \varphi)$ of simple complex Lie algebras $\mathfrak{s}$ and semi-simple automorphisms $\varphi$ such that $\mathfrak{s}^{\varphi}$ has rank 1 . Using the classification of semi-simple automorphisms of simple complex Lie algebras (compare [27, Chapter 3.3] and the references therein) it can be shown that the only possibilities for $\mathfrak{s}$ are $\mathfrak{s l}(2, \mathbb{C})$ and $\mathfrak{s l}(3, \mathbb{C})$. Then the claim follows from [20, Proposition 2.1] where the pairs of involutions on the classical Lie algebras are classified up to equivalence.

Remark 4.7. Let $U^{\mathbb{C}}=S \times \cdots \times S$ ( $k$ times) with $S=\operatorname{SL}(2, \mathbb{C})$, and let us consider the involutions $\sigma_{1}$ and $\sigma_{2}$ on $U^{\mathbb{C}}$ corresponding to $\left(\sigma_{1,1}, \theta\right)$ in the way described in Theorem 4.6. In this case we see that $\mathfrak{g}_{1} \cap \mathfrak{g}_{2}=: \mathfrak{t}_{0} \cong \mathfrak{s o}(2, \mathbb{R})$ is onedimensional and compact. Hence, the fundamental Cartan subset $C_{0}=\exp \left(i \mathrm{t}_{0}\right)$ is an exact slice for the $\left(G_{1} \times G_{2}\right)$-action on $U^{\mathbb{C}}$, i.e. every $\left(G_{1} \times G_{2}\right)$-orbit intersects $C_{0}$ in exactly one point. In particular, we conclude that each element $z \in U^{\mathbb{C}}$ is strongly regular and that $G_{1} \times G_{2}$ acts properly on $U^{\mathbb{C}}$.

In the following let us consider a point $z \in U^{\mathbb{C}}$ such that the orbit $M_{z}=$ $\left(G_{1} \times G_{2}\right) \cdot z$ is a closed hypersurface in $U^{\mathbb{C}}$. Without loss of generality we take $z$ to be of the form $z=n \exp (i \eta) \in C$ for some standard Cartan subset $C=n \exp (i \mathfrak{c})$. Because of $\operatorname{rk}\left(\mathfrak{g}_{1} \cap \mathfrak{g}_{2}\right)=1$ the Cartan subalgebra $\mathfrak{c} \subset \mathfrak{g}_{1} \cap \mathfrak{g}_{2}$ is one-dimensional and hence either $\mathfrak{c}=\mathfrak{t}$ is a maximal torus in $\mathfrak{k}_{1} \cap \mathfrak{k}_{2}$ or $\mathfrak{c}=\mathfrak{a}$ is a maximal Abelian subspace of $\mathfrak{p}_{1} \cap \mathfrak{p}_{2}$. Let

$$
\mathfrak{u}^{\mathbb{C}}=\bigoplus_{(\lambda, a) \in \tilde{\Lambda}} \mathfrak{u}_{\lambda, a}^{\mathbb{C}}
$$

be the extended weight space decomposition of $\mathfrak{u}^{\mathbb{C}}$ with respect to $\mathfrak{c}$.

Let us first assume that $\mathfrak{c}=\mathfrak{a}$ is non-compact. In this case every weight is real and we conclude from Theorem 3.15 that the only non-zero contributions to the Levi form of $M_{z}$ stem from the terms

$$
\mathcal{L}_{z}\left(\xi_{\lambda, a}, \xi_{-\lambda, a^{-1}}\right)=\frac{i}{a e^{-2 i \lambda(\eta)}-1}\left[\xi_{\lambda, a}, \xi_{-\lambda, a^{-1}}\right],
$$

where $\lambda \neq 0$ and $\xi_{\lambda, a}$ is a non-zero element in $\mathfrak{u}_{\lambda, a}^{\mathbb{C}}$ with $\sigma_{2}\left(\xi_{\lambda, a}\right)=\xi_{\lambda, a}$. Consequently, the restriction of the Levi form to

$$
\mathfrak{u}^{\mathbb{C}}[\lambda, a]=\mathfrak{u}_{\lambda, a}^{\mathbb{C}} \oplus \mathfrak{u}_{-\lambda, a^{-1}}^{\mathbb{C}}, \quad \lambda \in \Lambda^{+},
$$

has with respect to the bases $\left(\xi_{\lambda, a}, \xi_{-\lambda, a^{-1}}\right)$ of $\mathfrak{u}^{\mathbb{C}}[\lambda, a]$ and $\left[\xi_{\lambda, a}, \xi_{-\lambda, a^{-1}}\right]$ of $\mathfrak{a}$ the matrix

$$
\left(\begin{array}{cc}
0 & \frac{i}{a e^{-2 i \lambda(\eta)}-1} \\
-\frac{i}{a^{-1} e^{2 i \lambda(\eta)}-1} & 0
\end{array}\right),
$$


which has the eigenvalues $\pm \frac{1}{\left|a e^{-2 i \lambda(\eta)}-1\right|}$. Hence, we obtain a pair of one positive and one negative eigenvalue of the Levi form on $\mathfrak{u}^{\mathbb{C}}[\lambda, a]$ for each $\lambda \in \Lambda^{+}$.

If $\mathfrak{c}=\mathfrak{t}$ is compact, each weight is imaginary and we have to handle the cases $a= \pm 1$ and $a \neq \pm 1$ separately. If $a= \pm 1$, then $a=a^{-1}$ and consequently

$$
\mathfrak{u}^{\mathbb{C}}[\lambda, a]=\mathfrak{u}_{\lambda, a}^{\mathbb{C}} \oplus \mathfrak{u}_{-\lambda, a}^{\mathbb{C}}, \quad \lambda \in \Lambda^{+},
$$

holds. As basis of $\mathfrak{u}^{\mathbb{C}}[\lambda, a]$ we choose $\left(\xi_{\lambda, a}, \xi_{-\lambda, a}\right)$ with $\sigma_{2}\left(\xi_{\lambda, a}\right)=\xi_{-\lambda, a}$, and as basis of $\mathfrak{t}$ we take $i\left[\xi_{\lambda, a}, \xi_{-\lambda, a}\right]$. Then the Levi form has with respect to these bases the matrix

$$
\left(\begin{array}{cc}
\frac{1}{a e^{-2 i \lambda(\eta)}-1} & 0 \\
0 & -\frac{1}{a e^{2 i \lambda(\eta)}-1}
\end{array}\right) .
$$

If $a=1$, then both eigenvalues have the same sign, and if $a=-1$, the eigenvalues have different sign.

For $a \neq \pm 1$ we have

$$
\mathfrak{u}^{\mathbb{C}}[\lambda, a]=\mathfrak{u}_{\lambda, a}^{\mathbb{C}} \oplus \mathfrak{u}_{-\lambda, a^{-1}}^{\mathbb{C}} \oplus \mathfrak{u}_{\lambda, a^{-1}}^{\mathbb{C}} \oplus \mathfrak{u}_{-\lambda, a}^{\mathbb{C}}, \quad \lambda \in \Lambda^{+},
$$

and take $\left(\xi_{\lambda, a}, \xi_{-\lambda, a^{-1}}, \xi_{\lambda, a^{-1}}, \xi_{-\lambda, a}\right)$ as a basis of $\mathfrak{u}^{\mathbb{C}}[\lambda, a]$. Under the assumption $B_{\mathfrak{u}^{\mathbb{C}}}\left(\xi_{\lambda, a}, \xi_{-\lambda, a^{-1}}\right)=B_{\mathfrak{u}^{\mathbb{C}}}\left(\xi_{\lambda, a^{-1}}, \xi_{-\lambda, a}\right)$ we obtain $i\left[\xi_{\lambda, a}, \xi_{-\lambda, a^{-1}}\right]=i\left[\xi_{\lambda, a^{-1}}, \xi_{-\lambda, a}\right]$ which we take as a basis of $t$. With respect to these bases the restriction of the Levi form has the matrix

$$
\left(\begin{array}{cccc}
0 & \frac{1}{a e^{-2 i \lambda(\eta)}-1} & 0 & 0 \\
\frac{1}{a^{-1} e^{-2 i \lambda(\eta)}-1} & 0 & 0 & 0 \\
0 & 0 & 0 & \frac{1}{a^{-1} e^{-2 i \lambda(\eta)}-1} \\
0 & 0 & \frac{1}{a e^{-2 i \lambda(\eta)}-1} & 0
\end{array}\right)
$$

whose eigenvalues are given by $\pm \frac{1}{\left|a e^{-2 i \lambda(\eta)}-1\right|}$ and $\pm \frac{1}{\left|a^{-1} e^{-2 i \lambda(\eta)}-1\right|}$.

We summarize these results in the following

Theorem 4.8. If $\mathfrak{c}=\mathfrak{a}$ is non-compact, then each generic orbit $M_{z}$ with $z \in$ $n \exp (i \mathfrak{a})$ is Levi-q-convex with

$$
q=\# \Lambda^{+}
$$

If $\mathfrak{c}=\mathfrak{t}$ is compact, let us choose an ordering on the set of weights such that $\lambda(\eta)<0$ for all $\lambda \in \Lambda^{+}$and $z=n \exp (i \eta)$. Then each generic orbit $M_{z}$ with $z \in n \exp (i \mathfrak{t})$ is Levi-q-convex with

$$
q=\#\left\{(\lambda,-1) \in \widetilde{\Lambda} ; \lambda \in \Lambda^{+}\right\}+\#\left\{(\lambda, a) \in \widetilde{\Lambda} ; \lambda \in \Lambda^{+}, a \neq \pm 1\right\} .
$$


Moreover, this numbers for $q$ are sharp, i.e. $M_{z}$ is not Levi-q'-convex for any $q^{\prime}<q$.

Theorem 4.4 and Theorem 4.8 yield the following result.

Theorem 4.9. Let $M_{z}$ be a closed hypersurface orbit where $z \in C=n \exp (i \mathfrak{c})$ and let $\Omega$ be an invariant domain with $\partial \Omega=M_{z}$.

(1) If $\mathfrak{c}=\mathfrak{a}$ is non-compact, then $\Omega$ or $U^{\mathbb{C}} \backslash \bar{\Omega}$ is strictly $q$-complete with

$$
q=\# \Lambda^{+}
$$

and this $q$ is optimal.

(2) If $\mathfrak{c}=\mathfrak{t}$ is compact, then $\Omega$ or $U^{\mathbb{C}} \backslash \bar{\Omega}$ is strictly $q$-complete with

$$
q=\#\left\{(\lambda,-1) \in \tilde{\Lambda} ; \lambda \in \Lambda^{+}\right\}+\#\left\{(\lambda, a) \in \widetilde{\Lambda} ; \lambda \in \Lambda^{+}, a \neq \pm 1\right\}
$$

and this $q$ is optimal.

\subsection{The Levi form of invariant functions and $q$-complete domains}

Let us assume in this subsection that the intersection $\mathfrak{g}_{1} \cap \mathfrak{g}_{2}$ has arbitrary rank and contains a compact Cartan subalgebra $t$. For $z \in \exp (i \mathfrak{t})$, by Matsuki's result a generic orbit $M_{z}=\left(G_{1} \times G_{2}\right) \cdot z$ intersects the corresponding standard Cartan subset $C=\exp (i \mathfrak{t})$ in an orbit of the group $W_{K_{1} \times K_{2}}(C)$.

Remark 4.10. It can be shown that there exists a group isomorphism from

$$
W:=W_{K_{1} \cap K_{2}}(\mathfrak{t}):=\mathcal{N}_{K_{1} \cap K_{2}}(\mathfrak{t}) / \mathcal{Z}_{K_{1} \cap K_{2}}(\mathfrak{t})
$$

onto $W_{K_{1} \times K_{2}}(C)$ such that the diffeomorphism $\mathfrak{t} \rightarrow C, \eta \mapsto \exp (i \eta)$, intertwines the $W$-action on $t$ with the $W_{K_{1} \times K_{2}}(C)$-action on $C$ modulo this isomorphism [21].

We call a non-zero weight $\lambda \in \Lambda$ compact, if $(\lambda, 1) \in \widetilde{\Lambda}=\widetilde{\Lambda}\left(\mathfrak{u}^{\mathbb{C}}, \mathfrak{t}\right)$ and if $\lambda\left(\left[\xi_{\lambda, 1}, \sigma_{2}\left(\xi_{\lambda, 1}\right)\right]\right)<0$ for some $\xi_{\lambda, 1} \in \mathfrak{u}_{\lambda, 1}^{\mathbb{C}}$, and non-compact otherwise.

Remark 4.11. Let $(\lambda, 1) \in \widetilde{\Lambda}$ be given. Since $\mathfrak{t}$ is compact, the $\mathbb{C}$-linear involution $\sigma_{2} \theta$ stabilizes $\mathfrak{u}_{\lambda, 1}^{\mathbb{C}}$. Since this space is one-dimensional, it follows that $\sigma_{2} \theta= \pm \mathrm{id}$ holds on $\mathfrak{u}_{\lambda, 1}^{\mathbb{C}}$. Using the fact that after a suitable normalization $\lambda\left(\eta_{\lambda}\right)=2$ for $\eta_{\lambda}=-\left[\xi_{\lambda, 1}, \theta\left(\xi_{\lambda, 1}\right)\right]$ (Proposition 2.14) we conclude that $\lambda$ is compact if and only if $\sigma_{2}=\theta$ holds on $\mathfrak{u}_{\lambda, 1}^{\mathbb{C}} \oplus \mathfrak{u}_{-\lambda, 1}^{\mathbb{C}}$. Let $\mathfrak{s}_{\lambda}=\mathbb{C} \eta_{\lambda} \oplus \mathfrak{u}_{\lambda, 1}^{\mathbb{C}} \oplus \mathfrak{u}_{-\lambda, 1}^{\mathbb{C}} \cong \mathfrak{s l}(2, \mathbb{C})$. If $\lambda$ is compact, we have $\mathfrak{s}_{\lambda} \cap \mathfrak{g}_{2} \cong \mathfrak{s u}(2)$, while this intersection is isomorphic to $\mathfrak{s u}(1,1)$ for non-compact $\lambda$.

Let us assume that there exists a good ordering $\Lambda^{+}$on the set of non-zero weights, i.e. that that each positive non-compact weight is larger than every compact weight. This implies that the convex cone

$$
C_{\max }:=\left\{\eta \in \mathfrak{t} ; i \lambda(\eta) \geq 0 \text { for all non-compact } \lambda \in \Lambda^{+}\right\} \subset \mathfrak{t}
$$

is $W$-invariant. Let $\Omega:=G_{1} \exp \left(i C_{\max }^{0}\right) G_{2}$, where $C_{\max }^{0}$ is the interior of $C_{\max }$. 
Example 4.12. A sufficient condition for the existence of $\Omega$ is that the intersection $\mathfrak{g}_{1} \cap \mathfrak{g}_{2}$ is of Hermitian type.

Lemma 4.13. The group $G_{1} \times G_{2}$ acts properly on $\Omega$.

Proof. Using [25, Theorems 1.2.9 and 2.3.3] we only have to show that for all $z \in \Omega$ the isotropy $\left(G_{1} \times G_{2}\right)_{z}$ is compact. We can assume that $z$ lies in $\exp \left(i C_{\max }^{0}\right)$. Then $\left(G_{1} \times G_{2}\right)_{z}$ is a closed compatible subgroup of $U^{\mathbb{C}} \times U^{\mathbb{C}}$ and therefore it is enough to prove that $\mathfrak{g}_{2} \cap \operatorname{Ad}\left(z^{-1}\right) \mathfrak{g}_{1}$ is contained in $\mathfrak{u}$. If $z$ is strongly regular, then it follows that $\mathfrak{g}_{2} \cap \operatorname{Ad}\left(z^{-1}\right) \mathfrak{g}_{1}=\mathfrak{t}$ is contained in $\mathfrak{u}$. Therefore we have to consider non-regular points. By Theorem 3.6 the element $z=\exp (i \eta) \in \exp (i \mathfrak{t})$ is nonregular if and only if there is an extended weight $(\lambda, a) \neq(0,1)$ with $e^{2 i \lambda(\eta)}=a$. Since $\mathfrak{t}$ is compact, we have $\lambda(\eta) \in i \mathbb{R}$ and hence $a=e^{2 i \lambda(\eta)} \in S^{1} \cap \mathbb{R}^{>0}=\{1\}$, i.e. $a=1$ and $\lambda(\eta)=0$. Thus for a non-regular element $z=\exp (i \eta) \in \Omega$ only compact weights $(\lambda, 1)$ can appear in $\widetilde{\Lambda}(z)$, which implies

$$
\left(\mathfrak{g}_{2} \cap \operatorname{Ad}\left(z^{-1}\right) \mathfrak{g}_{1}\right)^{\mathbb{C}} \subset \mathfrak{t}^{\mathbb{C}} \oplus \underset{(\lambda, 1) \in \widetilde{\Lambda}: \lambda \text { compact }}{\bigoplus} \mathfrak{u}_{\lambda, 1}^{\mathbb{C}} .
$$

Since by the above remark $\sigma_{2}$ coincides with $\theta$ on $\mathfrak{u}_{\lambda, 1}^{\mathbb{C}}$ for compact $\lambda$, we conclude that $\mathfrak{g}_{2} \cap \operatorname{Ad}\left(z^{-1}\right) \mathfrak{g}_{1}$ is contained in $\mathfrak{u}$ which completes the proof.

It is known that the mapping $\mathcal{R}: \mathcal{C}^{\infty}(\Omega)^{G_{1} \times G_{2}} \rightarrow \mathcal{C}^{\infty}\left(C_{\max }^{0}\right)^{W}$,

$$
\Phi \mapsto \varphi: \eta \mapsto \Phi(\exp (i \eta)),
$$

is an isomorphism (compare [11]). The inverse $\mathcal{E}:=\mathcal{R}^{-1}$ is called the extension operator.

One would expect that the Levi form of an invariant smooth function on $\Omega$ is determined by the direction tangent to the $\left(G_{1} \times G_{2}\right)$-orbits and by a direction transversal to the orbit. The following lemma explains how the Levi form $L(\Phi)(z)$ is influenced by the complex tangent space of $\left(G_{1} \times G_{2}\right) \cdot z=M_{z}$.

Lemma 4.14. Let $\Phi \in \mathcal{C}^{\infty}(\Omega)^{G_{1} \times G_{2}}$ be given. If $v, w \in H_{z} M_{z} \subset T_{z} \Omega$, then we have

$$
L(\Phi)(z)(v, w)=-d^{c} \Phi(z) \mathcal{L}_{z}(v, w),
$$

where $\mathcal{L}_{z}$ is the Levi form of $M_{z}$.

Proof. By definition, the Levi form of $\Phi \in \mathcal{C}^{\infty}(\Omega)$ at the point $z \in \Omega$ is the Hermitian form $L(\Phi)(z)$ on $T_{z} \Omega$ associated to the $(1,1)$-form $\omega:=-\frac{1}{2} d d^{c} \Phi$. We use the formula

$$
d \omega(V, W)=V(\omega(W))-W(\omega(V))-\omega([V, W])
$$


and extend $v$ to a $\mathrm{CR}$ vector field $V$ on $M_{z}$ to compute as follows:

$$
\begin{aligned}
-d d^{c} \Phi(z)\left(v, J_{z} v\right) & =-v\left(d^{c} \Phi(J V)\right)+J_{z} v\left(d^{c} \Phi(V)\right)+d^{c} \Phi(z)[V, J V] \\
& =v(d \Phi(V))+J_{z} v(d \Phi(J V))+d^{c} \Phi(z)[V, J V] \\
& =v(V(\Phi))+J_{z} v(J V(\Phi))+d^{c} \Phi(z)[V, J V] .
\end{aligned}
$$

Since the vector fields $V$ and $J V$ are tangent to the orbit and since $\Phi$ is constant along the orbit, we obtain

$$
L(\Phi)(z)(v, v)=-\frac{1}{2} d d^{c} \Phi(z)(v, J v)=\frac{1}{2} d \Phi(z) J[V, J V]_{z}=-d \Phi(z) J \widehat{\mathcal{L}}_{z}(v) .
$$

Thus the claim follows from the polarization identities.

Proposition 4.15. Let $z \in \Omega$ and let $T_{z} \Omega=T_{z} U^{\mathbb{C}}$ be identified with

$$
\mathfrak{u}^{\mathbb{C}}=\bigoplus_{(\lambda, a) \in \tilde{\Lambda}} \mathfrak{u}_{\lambda, a}^{\mathbb{C}}
$$

via $\left(\ell_{z}\right)_{*}$. Let $\varphi \in \mathcal{C}^{\infty}\left(C_{\max }^{0}\right)^{W}$ be given and let $\Phi:=\mathcal{E}(\varphi)$ be its extension to a smooth $\left(G_{1} \times G_{2}\right)$-invariant function on $\Omega$. Then the decomposition (4.1) is orthogonal with respect to the Levi form $L(\Phi)(z)$.

Proof. First we assume that $z \in \Omega \cap U_{s r}^{\mathbb{C}}$ holds. In view of Lemma 4.14 it is enough to show that $\mathfrak{t}^{\mathbb{C}}$ and $H_{z} M_{z} \cong \bigoplus_{(\lambda, a) \neq(0,1)} \mathfrak{u}_{\lambda, a}^{\mathbb{C}}$ are orthogonal with respect to $L(\Phi)(z)$. Thus let $v \in \mathfrak{t}$ and $w \in \mathfrak{u}_{\lambda, a}^{\mathbb{C}}$ be given. Since $J v$ and $w$ are tangent to $M_{z}=\left(G_{1} \times G_{2}\right) \cdot z$, there are elements $\eta, \xi \in \mathfrak{q}_{z} \subset \mathfrak{g}_{1} \oplus \mathfrak{g}_{2}$ such that $J v=\eta_{\Omega}(z)$ and $w=\xi_{\Omega}(z)$ hold, where $\eta_{\Omega}$ and $\xi_{\Omega}$ are the corresponding vector fields on $\Omega$. Using the same arguments as in the proof of Lemma 4.14 together with the invariance of $\Phi$ we obtain

$$
L(\Phi)(z)(v, w)=d^{c} \Phi(z)\left[\eta_{\Omega}, \xi_{\Omega}\right](z)-i d^{c} \Phi(z)\left[\eta_{\Omega}, \xi_{\Omega}\right](z)
$$

Since $\left[\eta_{\Omega}, \xi_{\Omega}\right](z)=[\eta, \xi]_{\Omega}(z) \in H_{z} M_{z}$, the invariance of $\Phi$ implies

$$
d^{c} \Phi(z)\left[\eta_{\Omega}, \xi_{\Omega}\right](z)=0,
$$

which was to be shown.

Finally, if $z \in \Omega$ is not contained in $U_{s r}^{\mathbb{C}}$, we can still compute the Levi form separately on each $\mathfrak{u}^{\mathbb{C}}[\lambda, a]$ which appears in the tangent space of $\left(G_{1} \times G_{2}\right) \cdot z$ because $L(\Phi)(z)$ depends continuously on $z$.

We will apply Proposition 4.15 in order to establish existence of a strictly $q$ pseudo-convex exhaustion function on $\Omega$. The following theorem extends Neeb's result on open complex Ol'shanskiı̆ semi-groups to the case $G_{1} \neq G_{2}$. 
Theorem 4.16. The domain $\Omega$ is q-complete for

$$
n-q=\operatorname{rk}\left(\mathfrak{g}_{1} \cap \mathfrak{g}_{2}\right)+\#\{(\lambda, a) \in \widetilde{\Lambda} ; a=1\} .
$$

Proof. In the first step we decompose the convex cone $C_{\max }$ into its maximal linear subspace and a pointed convex cone. More precisely, let $H\left(C_{\max }\right):=C_{\max } \cap$ $\left(-C_{\max }\right)$ be the maximal linear subspace of $C_{\max }$; since $C_{\max }$ is polyhedral, elementary considerations show that we can write $C_{\max }$ as $C_{\max }=\widetilde{C}_{\max } \oplus H\left(C_{\max }\right)$ where $\widetilde{C}_{\max }$ is a $W$-invariant pointed convex cone in a $W$-invariant complement of $H\left(C_{\max }\right)$ in $\mathfrak{t}$ (see for example [15], Remark 1.25).

Let $\psi \in \mathcal{C}^{\infty}\left(H\left(C_{\max }\right)\right)^{W}$ be a strictly convex positive exhaustion function and let $\chi$ be the characteristic function of $\widetilde{C}_{\max }$ (see e.g. [24], Chapter V.5, for the definition). We need the following properties of $\chi$ which are proven in [24, Theorem V.5.4]:

(i) $\chi$ is positive, strictly convex and analytic on $\widetilde{C}_{\max }^{0}$;

(ii) $\chi$ is $W$-invariant (since $|\operatorname{det} w|=1$ for all $w \in W$ );

(iii) if $x_{n} \rightarrow x \in \partial \widetilde{C}_{\max }$, then $\chi\left(x_{n}\right) \rightarrow \infty$;

(iv) the mapping $C_{\max }^{0} \rightarrow\left(C_{\max }^{*}\right)^{0}, x \mapsto-d(\log \chi)(x)$, is bijective; by [22, Lemma I.11] this means that the function $\log \chi$ (and hence $\chi$ ) is $C_{\max }$-decreasing.

Combining the functions $\chi$ and $\psi$ we obtain the function

$$
\varphi: C_{\max }^{0} \rightarrow \mathbb{R}^{>0}, \quad \varphi(x+y)=\chi(x)+\psi(y) \quad\left(x \in \widetilde{C}_{\max }^{0}, y \in H\left(C_{\max }\right)\right) .
$$

We conclude that $\varphi$ is a smooth strictly convex function with $\varphi\left(x_{n}\right) \rightarrow \infty$ whenever $x_{n} \rightarrow x \in \partial C_{\max }$.

Let $\Phi:=\mathcal{E}(\varphi)$ be the corresponding smooth $\left(G_{1} \times G_{2}\right)$-invariant function in $\Omega$. Since $G_{1} \times G_{2}$ acts by holomorphic transformations, it is sufficient to compute the Levi form of $\Phi$ at points $z=\exp (i \eta) \in \exp \left(i C_{\max }^{0}\right)$ where $\eta \in C_{\max }^{0}$. Due to Proposition 4.15 we may compute the Levi form $L(\Phi)(z)$ on each $\mathfrak{u}^{\mathbb{C}}[\lambda, a]$ separately where again we identify $T_{z} \Omega$ with $\mathfrak{u}^{\mathbb{C}}$.

Since $\varphi$ is strictly convex, its extension $\Phi$ is strictly plurisubharmonic on $\Omega \cap$ $\exp \left(\mathfrak{t}^{\mathbb{C}}\right)$. Hence, we obtain for every $z \in \Omega$ at least $\operatorname{dim} \mathfrak{t}=\operatorname{rk}\left(\mathfrak{g}_{1} \cap \mathfrak{g}_{2}\right)$ positive eigenvalues of $L(\Phi)(z)$.

Next, we consider generalized weights of the form $(\lambda, 1) \in \widetilde{\Lambda}$. We have $\mathfrak{u}^{\mathbb{C}}[\lambda, 1]=\mathfrak{u}_{\lambda, 1}^{\mathbb{C}} \oplus \mathfrak{u}_{-\lambda, 1}^{\mathbb{C}}$ and set $\mathfrak{s}_{\lambda}:=\mathbb{C} \eta_{\lambda} \oplus \mathfrak{u}^{\mathbb{C}}[\lambda, 1] \cong \mathfrak{s l}(2, \mathbb{C})$. Moreover, we write $\eta=\eta_{\lambda}+\left(\eta-\eta_{\lambda}\right)$ and consequently have $z=\exp \left(i\left(\eta-\eta_{\lambda}\right)\right) \exp \left(\eta_{\lambda}\right)$. Since left multiplication with $\exp \left(i\left(\eta-\eta_{\lambda}\right)\right)$ is holomorphic, we can reduce the computation of $L(\Phi)(z)$ to the case of functions on $S_{\lambda} \cong \operatorname{SL}(2, \mathbb{C})$ which are invariant under $\mathrm{SU}(2) \times \mathrm{SU}(2)$ (when $\lambda$ is compact) or to functions on the open Ol'shanskii semi-group in $\operatorname{SL}(2, \mathbb{C})$ invariant under $\operatorname{SU}(1,1) \times \operatorname{SU}(1,1)$ (when $\lambda$ is non-compact).

We will show by direct computations that $L(\Phi)(z)$ is positive on each $\mathfrak{u}^{\mathbb{C}}[\lambda, 1]$. To do this we start with the case that $\lambda$ is compact. As remarked above, we can 
reduce the computations to the case that $U^{\mathbb{C}}=S_{\lambda}=\operatorname{SL}(2, \mathbb{C})$ and $G_{1} \times G_{2}=$ $\mathrm{SU}(2) \times \mathrm{SU}(2)$. In this case

$$
\mathfrak{t}:=\left\{\eta_{t}:=\left(\begin{array}{cc}
i t & 0 \\
0 & -i t
\end{array}\right) ; t \in \mathbb{R}\right\}
$$

is a maximal torus in $\mathfrak{s u}(2)$, the set of roots is given by $\Delta=\{ \pm \alpha\}$ where $\alpha\left(\eta_{t}\right)=$ $2 i t$, and $\mathfrak{u}_{\alpha}^{\mathbb{C}}=\mathbb{C}\left(\begin{array}{ll}0 & 1 \\ 0 & 0\end{array}\right)$. The Weyl group of $\mathfrak{t}$ acts by the reflection $\eta_{t} \mapsto \eta_{-t}=$ $-\eta_{t}$. We may assume that the base point $z_{0}$ of the $\left(G_{1} \times G_{2}\right)$-orbit is of the form $z_{0}=\exp \left(i \eta_{t_{0}}\right)$ with $t_{0}>0$. Next, we describe the intrinsic Levi form $\mathcal{L}_{z_{0}}$ on $\mathfrak{u}^{\mathbb{C}}[\alpha]=\mathfrak{u}_{\alpha}^{\mathbb{C}} \oplus \mathfrak{u}_{-\alpha}^{\mathbb{C}}$. By Theorem 3.15 we have $\mathfrak{u}_{\alpha}^{\mathbb{C}} \perp \mathfrak{u}_{-\alpha}^{\mathbb{C}}$ with respect to $\mathcal{L}_{z_{0}}$ and

$$
\begin{aligned}
\mathcal{L}_{z_{0}}\left(\xi_{\alpha}, \xi_{\alpha}\right)=\frac{i}{e^{-2 i \alpha\left(\eta_{t_{0}}\right)}-1}\left[\xi_{\alpha}, \theta\left(\xi_{\alpha}\right)\right] & =-\frac{1}{e^{4 t_{0}}-1} i \eta_{\alpha} \in \mathfrak{t} \quad \text { as well as } \\
\mathcal{L}_{z_{0}}\left(\xi_{-\alpha}, \xi_{-\alpha}\right) & =\frac{i}{e^{2 i \alpha\left(\eta_{t_{0}}\right)}-1}\left[\theta\left(\xi_{\alpha}\right), \xi_{\alpha}\right]=\frac{1}{e^{-4 t_{0}}-1} i \eta_{\alpha} \in \mathfrak{t},
\end{aligned}
$$

where $\xi_{\alpha}=\left(\begin{array}{ll}0 & 1 \\ 0 & 0\end{array}\right)$ and $\xi_{-\alpha}=\theta\left(\xi_{\alpha}\right)$ hold. In particular we have $i \eta_{\alpha}=\left(\begin{array}{cc}i & 0 \\ 0 & -i\end{array}\right)$. Since $t_{0}$ is positive, the coefficients in front of $i \eta_{\alpha}$ are in both cases negative. In the rest of the proof we identify $\mathfrak{t}$ with the real real line $\mathbb{R}$ by mapping $i \eta_{\alpha}$ to 1 . Under the identification $\mathfrak{t} \cong \mathbb{R}$ we view $\varphi$ as a strictly convex function $\mathbb{R} \rightarrow \mathbb{R} \geq 0$. Since $\varphi(t)=\varphi(-t)$ holds by $W$-invariance, elementary arguments show that $\varphi$ must have a minimum in $0 \in \mathbb{R}$ and must be monotonely increasing on $\mathbb{R}^{>0}$ and monotonely decreasing on $\mathbb{R}^{<0}$. This observation proves

$$
-d \Phi\left(z_{0}\right) J \widehat{\mathcal{L}}_{z_{0}}\left(\xi_{\alpha}\right)=-\varphi^{\prime}\left(t_{0}\right) \frac{-1}{e^{4 t_{0}}-1}>0
$$

and

$$
-d \Phi\left(z_{0}\right) J \widehat{\mathcal{L}}_{z_{0}}\left(\xi_{-\alpha}\right)=-\varphi^{\prime}\left(t_{0}\right) \frac{1}{e^{-4 t_{0}}-1}>0 .
$$

The fact that $L(\Phi)(z)$ is positive on $\mathfrak{u}^{\mathbb{C}}[\lambda, 1]$ can be viewed as a special case of the theorem of Azad and Loeb that $W$-invariant strictly convex functions on a maximal torus in $\mathfrak{s u}(2)$ extend to strictly plurisubharmonic functions on $\mathrm{SL}(2, \mathbb{C})$.

If $\lambda$ is non-compact, then we may assume that $U^{\mathbb{C}}=S_{\lambda}=\operatorname{SL}(2, \mathbb{C})$ and $G_{1} \times G_{2}=\mathrm{SU}(1,1) \times \mathrm{SU}(1,1)$. The subspace $\mathfrak{t}=\mathbb{R}\left(\begin{array}{cc}i & 0 \\ 0 & -i\end{array}\right)$ is a compact Cartan subalgebra of $\mathfrak{g}=\mathfrak{s u}(1,1)$ which gives the same root system as above. Choosing the ordering such that the root $\alpha$ is positive, we obtain

$$
C_{\max } \cap \mathfrak{s}_{\lambda}=\left\{\eta_{t}=\left(\begin{array}{cc}
i t & 0 \\
0 & -i t
\end{array}\right) ; i \alpha\left(\eta_{t}\right)=-2 t \geq 0\right\}=\left\{\eta_{t} ; t \leq 0\right\} .
$$

Hence, if $t_{0}<0$, then $z_{0}=\exp \left(i \eta_{t_{0}}\right)$ lies in $\Omega \cap U_{s r}^{\mathbb{C}}$. By Theorem 3.15 we have

$$
\begin{gathered}
\mathcal{L}_{z_{0}}\left(\xi_{\alpha}, \xi_{\alpha}\right)=\frac{i}{e^{-2 i \alpha\left(\eta_{t_{0}}\right)}-1}\left[\xi_{\alpha}, \sigma\left(\xi_{\alpha}\right)\right]=\frac{1}{e^{4 t_{0}}-1} i \eta_{\alpha} \in C_{\max }^{0} \quad \text { and } \\
\mathcal{L}_{z_{0}}\left(\xi_{-\alpha}, \xi_{-\alpha}\right)=\frac{i}{e^{2 i \alpha\left(\eta_{t_{0}}\right)}-1}\left[\sigma\left(\xi_{\alpha}\right), \xi_{\alpha}\right]=-\frac{1}{e^{-4 t_{0}}-1} i \eta_{\alpha} \in C_{\max }^{0},
\end{gathered}
$$


where $\xi_{\alpha}=\left(\begin{array}{ll}0 & 1 \\ 0 & 0\end{array}\right)$ and $\xi_{-\alpha}=\left(\begin{array}{ll}0 & 0 \\ 1 & 0\end{array}\right)$ hold. Note that the coefficients in front of $i \eta_{\alpha}$ are negative. Again we identify $\mathfrak{t}$ with $\mathbb{R}$ via $i \eta_{\alpha} \leftrightarrow 1$. Under this identification $C_{\max }^{0} \cap \mathfrak{s}_{\lambda}$ is mapped onto $(-\infty, 0)$ since $i \eta_{\alpha}=\left(\begin{array}{cc}i & 0 \\ 0 & -i\end{array}\right) \notin C_{\max }^{0} \cap \mathfrak{s}_{\lambda}$ holds. The characteristic function $\chi$ restricts to a monotonely increasing strictly convex function $\widehat{\chi}:(-\infty, 0) \rightarrow \mathbb{R}^{\geq 0}$ with the property $\widehat{\chi}(t) \rightarrow \infty$ for $t \rightarrow 0$. This implies

$$
-d \Phi\left(z_{0}\right) J \widehat{\mathcal{L}}_{z_{0}}\left(\xi_{\alpha}\right)=-\widehat{\chi}^{\prime}\left(t_{0}\right) \frac{1}{e^{4 t_{0}}-1}>0
$$

and

$$
-d \Phi\left(z_{0}\right) J \widehat{\mathcal{L}}_{z_{0}}\left(\xi_{-\alpha}\right)=-\widehat{\chi}^{\prime}\left(t_{0}\right) \frac{-1}{e^{-4 t_{0}}-1}>0,
$$

i.e. we obtain two positive eigenvalues of the Levi form $L(\Phi)$.

Hence, we conclude that the extension $\Phi$ is strictly $q$-pseudo-convex for

$$
n-q=\operatorname{dim} \mathfrak{t}^{\mathbb{C}}+\#\{(\lambda, 1) \in \widetilde{\Lambda} ; \lambda \text { compact }\}+\#\{(\lambda, 1) \in \widetilde{\Lambda} ; \lambda \text { non-compact }\} .
$$

If $z_{n} \rightarrow z \in \partial \Omega$, then $\Phi\left(z_{n}\right) \rightarrow \infty$ holds by construction and hence we conclude that $\Omega$ is $q$-complete for the above $q$.

Remark 4.17. The fact that each $(\lambda, 1) \in \widetilde{\Lambda}$ yields positive eigenvalues for the Levi form of $\Phi$ can also be deduced from [24, Corollary XIII.5.17] if $\mathfrak{g}_{1} \cap \mathfrak{g}_{2}$ is of Hermitian type since we have $\left(\mathfrak{g}_{1} \cap \mathfrak{g}_{2}\right)^{\mathbb{C}}=\mathfrak{t}^{\mathbb{C}} \oplus \bigoplus_{(\lambda, 1) \in \widetilde{\Lambda}} \mathfrak{u}_{\lambda, 1}^{\mathbb{C}}$ and since the characteristic function $\chi$ of $C_{\max }$ is strictly convex and $C_{\max }$-decreasing.

\section{References}

[1] A. ANDREOTTI and H. GRAUERT, Théorème de finitude pour la cohomologie des espaces complexes, Bull. Soc. Math. France 90 (1962), 193-259.

[2] H. AZAD and J.-J. LOEB, Plurisubharmonic functions and Kählerian metrics on complexification of symmetric spaces, Indag. Math. (N.S.) 3 (1992), 365-375.

[3] M. Salah Baouendi, P. Ebenfelt and L. Preiss Rothschild, "Real Submanifolds in Complex Space and their Mappings", Princeton Mathematical Series, Vol. 47, Princeton University Press, Princeton, NJ, 1999.

[4] A. Boggess, "CR Manifolds and the Tangential Cauchy-Riemann complex", Studies in Advanced Mathematics, CRC Press, Boca Raton, FL, 1991.

[5] R. J. BREMIGAN, Invariant analytic domains in complex semisimple groups, Transform. Groups 1 (1996), 279-305.

[6] C. Chevalley, "Theory of Lie Groups. I", Princeton University Press, Princeton, N. J., 1946 [Eighth Printing, 1970].

[7] J.-P. DEMAILLY, "Complex Analytic and Algebraic Geometry", available at http://wwwfourier.ujf-grenoble.fr/ demailly/books.html.

[8] F. DOCQUIER and H. GRAUERT, Levisches Problem und Rungescher Satz für Teilgebiete Steinscher Mannigfaltigkeiten, Math. Ann. 140 (1960), 94-123.

[9] M. G. EASTWOOD and G. Vigna SURIA, Cohomologically complete and pseudoconvex domains, Comment. Math. Helv. 55 (1980), 413-426.

[10] G. FELS and L. GEATTI, Geometry of biinvariant subsets of complex semisimple Lie groups, Ann. Scuola Norm. Sup. Pisa Cl. Sci. (4) 26 (1998), 329-356. 
[11] M. Flensted-Jensen, Spherical functions of a real semisimple Lie group. A method of reduction to the complex case, J. Funct. Anal. 30 (1978), 106-146.

[12] J. HilgerT and K.-H. NeEB, "Lie Semigroups and their Applications", Lecture Notes in Mathematics, Vol. 1552, Springer-Verlag, Berlin, 1993.

[13] P. HeInZNER and G. W. SChWARZ, Cartan decomposition of the moment map, Math. Ann. 337 (2007), 197-232.

[14] J. E. Humphreys, "Conjugacy Classes in Semisimple Algebraic Groups", Mathematical Surveys and Monographs, Vol. 43, American Mathematical Society, Providence, RI, 1995.

[15] L. KAUP, "Vorlesungen über Torische Varietäten", Konstanzer Schriften in Mathematik und Informatik, Nr. 130, Fassung vom Herbst 2001.

[16] S. Kobayashi and K. Nomizu, "Foundations of Differential Geometry. Vol I", Interscience Publishers, a division of John Wiley \& Sons, New York-London, 1963.

[17] A. W. KNAPP, "Lie Groups Beyond an Introduction", second ed., Progress in Mathematics, Vol. 140, Birkhäuser Boston Inc., Boston, MA, 2002.

[18] M. LASSALle, Sur la transformation de Fourier-Laurent dans un groupe analytique complexe réductif, Ann. Inst. Fourier (Grenoble) 28 (1978), 115-138.

[19] T. MATSUKI, Double coset decompositions of reductive Lie groups arising from two involutions, J. Algebra 197 (1997), 49-91.

[20] T. MATSUKI, Classification of two involutions on compact semisimple Lie groups and root systems, J. Lie Theory 12 (2002), 41-68.

[21] C. MiEBACH, Geometry of invariant domains in complex semi-simple Lie groups, Dissertation, Bochum, 2007.

[22] K.-H. NeEB, Invariant convex sets and functions in Lie algebras, Semigroup Forum $\mathbf{5 3}$ (1996), 230-261.

[23] K.-H. NEEB, On the complex and convex geometry of Ol'shanskiŭ semigroups, Ann. Inst. Fourier (Grenoble) 48 (1998), 149-203.

[24] K.-H. NeEB, "Holomorphy and Convexity in Lie Theory", de Gruyter Expositions in Mathematics, Vol. 28, Walter de Gruyter \& Co., Berlin, 2000.

[25] R. S. PALAIS, On the existence of slices for actions of non-compact Lie groups, Ann. of Math. (2) 73 (1961), 295-323.

[26] R. Steinberg, "Endomorphisms of Linear Algebraic Groups", Memoirs of the American Mathematical Society, No. 80, American Mathematical Society, Providence, R.I., 1968.

[27] È. B. VinBerg (ed.), "Lie Groups and Lie Algebras, III", Encyclopaedia of Mathematical Sciences, Vol. 41, Springer-Verlag, Berlin, 1994.

Fakultät für Mathematik

Ruhr-Universität Bochum

Universitätsstraße 150

D - 44780 Bochum, Germany

christian.miebach@ruhr-uni-bochum.de 